\title{
The Fascinating World of Phosphanylphosphonates: From Acetylenic Phosphaalkenes to Reductive Aldehyde Couplings
}

\author{
Juri Mai \\ Sascha Ott* (D) \\ Department of Chemistry, Ångström Laboratory, Uppsala University, \\ Box 523, 75120 Uppsala, Sweden \\ sascha.ott@kemi.uu.se \\ Dedicated to Professor Rüdiger Faust
}

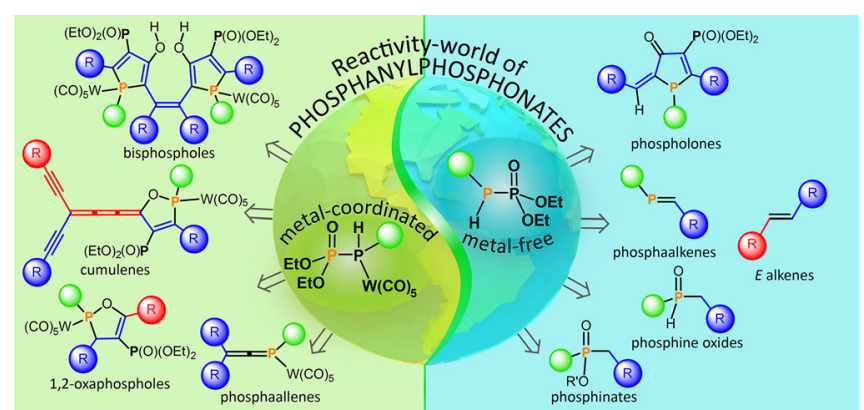

\section{Combining Acetylenes with Phospha- alkenes}

Our group's journey into the chemistry of phosphanylphosphonates started a bit more than ten years ago with our first grant from the Swedish Research Council for a study on acetylenic phosphaalkenes, i.e. the heavier congeners of ethynylethenes. The project was strongly inspired by the works of François Diederich on conjugated oligoenynes based on the 1,2-diethynylethene unit 1 (hex-3ene-1,5-diyne, DEE) which exists as three constitutional isomers: $(E)-\mathbf{1},(Z)-\mathbf{1}$, and gem-1 (Figure 1$){ }^{1}{ }^{1}$ The $(Z)$-isomers of these enediynes have attracted wide interest for their use as potential antiviral and antitumor agents in medicinal chemistry. This is mainly due to their ability to undergo a Bergman cyclization to give biologically active aromatic 1,4-diradical systems that are able to cleave DNA. ${ }^{2}$ The DEE units are also important synthetic modules for further extension to the larger tetraethynylethene 2 [3,4-diethynylhex-3-ene-1,5-diyne (TEE); Figure 1] and for the construction of conjugated systems through acetylenic coupling reactions. ${ }^{1,3}$
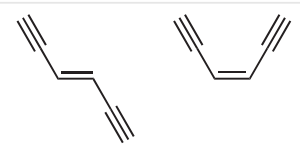

(E)-DEE 1

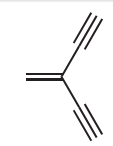

(Z)-DEE 1

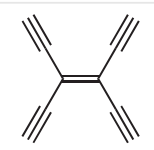

TEE 2
$4 \quad$ Reactions with Acetylenic Ketones

4.1 Metal-Coordinated Phosphanylphosphonate and Monoacetylenic Ketones

4.2 Metal-Coordinated Phosphanylphosphonate and Diacetylenic Ketones

4.3 Metal-Free Phosphanylphosphonate and Diacetylenic Ketones

5 Metal-Free Phosphanylphosphonate as a Coupling Reagent for Aldehydes

$6 \quad$ E-Alkenes by the Reductive Coupling of Two Aldehydes

7 Conclusions and Outlook

Key words acetylenic phosphaalkenes, phosphanylphosphonates, bisphospholes, oxaphospholes, phospholones, alkenes
Figure 1 Diethynylethenes (DEEs) and tetraethynylethene (TEE) as building blocks for conjugated oligoenynes ${ }^{1}$

Such fully $\pi$-conjugated systems consisting of ethylene, acetylene, or arene entities are often referred to as acetylenic scaffolding ${ }^{4}$ and they have various applications in the emerging fields of organic and molecular electronics and photonics. ${ }^{3,5}$ These acetylenes provide a conducting back- 
bone that can connect various functional units in, for example, molecular wires, diodes, switches, charge- or energytransfer systems, and other components for molecular electronics, nonlinear optics, organic conductors, polyelectrochromic materials, or organic light-emitting diodes. ${ }^{6}$ The electronic and material properties of the conjugated scaffolds depend on the type of conjugation - linear as in $(E / Z)$ $\mathbf{1}$ or cross-conjugated as in gem-1 - and on further functionalization with aryl donor-acceptor groups. ${ }^{5 \mathrm{~b}}$

The motivation to incorporate phosphorus into the diethynylethene units is based on the diagonal relationship between carbon and low-coordinate phosphorus, as '...carbon is more similar to its diagonal relative, phosphorus, than to silicon'. ${ }^{7}$ This 'Phosphorus: The Carbon Copy' relationship is particularly strong in low-coordinate phosphorus compounds with coordination numbers of one or two, i.e., when phosphorus is engaged in $\pi$-bonds or aromatic systems. ${ }^{8}$ In comparison to ethylenes, phosphaalkenes have an intrinsic dipole moment (Figure 2) and are polar molecules due to the difference in electronegativity between phosphorus $\left(\chi_{\mathrm{P}}=2.19\right)$ and carbon $\left(\chi_{\mathrm{C}}=2.55\right) .{ }^{9}$ Although the $\sigma$ component of the $\mathrm{P}=\mathrm{C}$ bond is polarized $\left(\mathrm{P}^{\delta+}-\mathrm{C}^{\delta-}\right)$, the effective $\pi$-electronegativities are nearly the same. ${ }^{10}$ In addition, owing to the slightly higher HOMO energy level of the parent phosphaalkene $\left({ }^{\pi} \mathrm{C}=\mathrm{P}\right.$ : $\left.-10.30 \mathrm{eV},{ }^{n} \mathrm{P}:-10.70 \mathrm{eV}\right) \mathrm{com}-$ pared with that of ethylene $(-10.51 \mathrm{eV})$, the chemical reactivity of compounds containing a $\mathrm{P}=\mathrm{C}$ unit is slightly higher than, although still rather close to, that of olefins. ${ }^{8}$

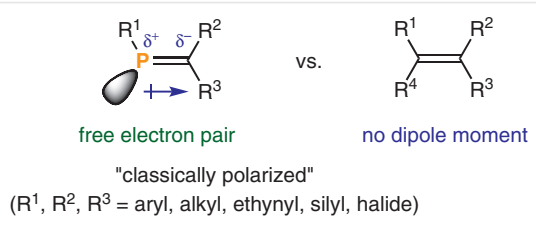

Figure 2 Structural comparison of phosphaethylene with ethylene

Along these lines, numerous standard reactions of the $\mathrm{C}=\mathrm{C}$ unit, such as 1,2-additions, epoxidations, oligomerizations, polymerizations, $E / Z$ photoisomerizations, and $[2+n]-$ cycloadditions, as well as pericyclic reactions, such as the phospha-Cope rearrangement, have been reported for phosphaalkenes. $^{7-8,11}$

At the start of our project about ten years ago, phosphaalkenes had appeared only very sporadically in the context of acetylene scaffolding, and the combination of acetylenes with phosphaalkenes seemed a rich area for explorations. In particular, P-inclusion leads to perturbations of electronic properties and is predicted to lower the HOMOLUMO gaps of the entire $\pi$-conjugate. ${ }^{12}$ It also gives a possibility for further postsynthetic modifications of the optical and electronic properties and for communication through the $\pi$-conjugated backbone. This can be achieved by oxidation $\left(\mathrm{O}, \mathrm{S}\right.$, or Se) of the P-center, ${ }^{7-8,11 a, f}$ by coordination with a Lewis acid to the phosphorus lone pair, ${ }^{13}$ or by tuning of the polarity of the $\mathrm{P}=\mathrm{C}$ double bond. ${ }^{14}$ In this context, the inclusion of phosphorus into larger $\pi$-conjugates has been

\section{Biographical Sketches}
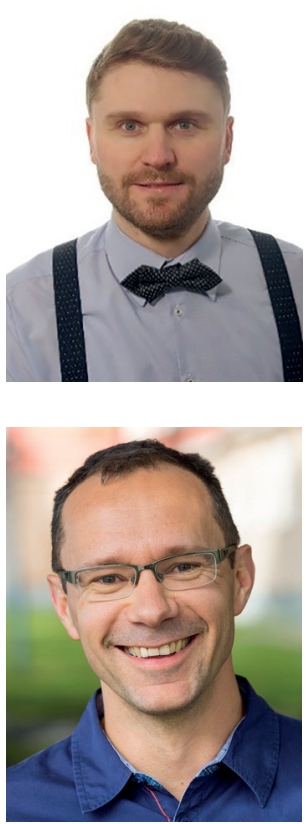

Juri Mai was born in the Ukraine in 1987. He obtained his diploma (Dipl.-NanoSc.) degree in nanostructure and molecular sciences from the University of Kassel, Germany, in 2014. During his diploma thesis in the group of Prof. Dr. Rüdi-

Sascha Ott is Professor in Synthetic Molecular Chemistry at the Department of ChemistryÅngström Laboratory of Uppsala University, Sweden. His research interests lie at the interface between organic and molecular inorganic chemistry. ger Faust, he worked on the synthesis and characterization of novel octaphenylphthalocyanines. Following his interest in organic synthesis, he then joined the research group of Prof. Dr. Sascha Ott at Uppsala University, Sweden, where he

The group's research portfolio spans from the inclusion of lowcoordinate phosphorus into $\pi$ conjugates and the use of phosphaorganic compounds as reagents in organic transformations, to the development of molecular catalysts completed his Ph.D. at the beginning of 2019. His doctoral studies mainly focused on method development for the formation of olefins by reductive coupling of two aldehydes through organophosphorus chemistry.

of energy relevance. He currently holds a consolidator grant from the European Research Council on molecular redox catalysis in metal-organic frameworks. 
coined as the phosphorus 'doping effect'. ${ }^{15}$ All these features open opportunities that are not presented by classical all-carbon-based designs.

With these ideas in mind, we decided to fuse the separated fields of phosphaalkenes and oligoacetylenes and to investigate the chemistry of acetylenic phosphaalkenes (APAs). At that time, such molecular systems were synthetically completely unexplored, and our works represented the first examples of heteroatom-containing oligoacetylenes. In analogy to their all-carbon congeners, various isomeric forms are possible (Figure 3 ). (E)- and (Z)-P,C-diacetylenic phosphaalkenes $3\left(P, C-\mathrm{A}_{2} \mathrm{PA}\right)$, which have one acetylene group on the phosphorus and one at the carbon atom, and the geminal $C, C$-diacetylenic phosphaalkene $4\left(C, C-A_{2}-\right.$ $\mathrm{PA})$ are heavier congeners of the corresponding DEEs in Figure 1 , whereas peracetylenic phosphaalkene $\mathbf{5}\left(P, C, C-\mathrm{A}_{3} \mathrm{PA}\right)$ is related to TEE.

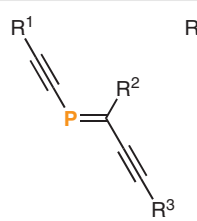

(E)-3

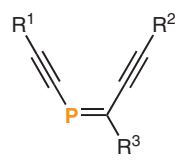

$(Z)-3$

$P, C$-diacetylenic phosphaalkenes

$P, C-\mathrm{A}_{2} \mathrm{PA}$

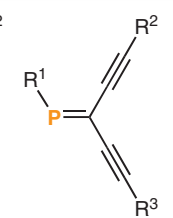

4

C, C-diacetylenic peracetylenic phosphaalkenes phosphaalkenes

$C, C-\mathrm{A}_{2} \mathrm{PA}$

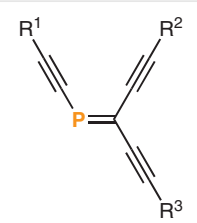

5

$P, C, C-\mathrm{A}_{3} \mathrm{PA}$
Figure 3 Various types of acetylenic phosphaalkenes (APAs)

\section{Synthetic Examples of Acetylenic Phos- phaalkenes}

Our first synthetic steps to introduce phosphaalkenes into the field of oligoacetylenes were taken in $2008 .{ }^{16} \mathrm{Be}-$ fore this, only a few examples of monoacetylenic $P$ - and $C$ APAs had been reported in the literature. ${ }^{17}$ These compounds were prepared by treatment of $P$ - or $C$-halogenated phosphaalkenes, respectively, with acetylene-containing Grignard reagents.

In planning our synthetic strategy for the APAs, it was essential to consider stability issues to prevent oligomerizations, polymerizations, or side reactions towards small molecules. The classical approaches to ensure stability are thermodynamic and kinetic stabilizations ${ }^{18}$ or, alternatively, coordination of metal fragments to the lone pair of the phosphaalkene. ${ }^{13}$ Whereas thermodynamic stabilization can be achieved by including the $\mathrm{P}=\mathrm{C}$ bond in an aromatic system, as in phosphinines, kinetic stabilization entails the use of bulky substituents on the phosphorus heteroatom. From the large variety of such bulky groups, the most common is the 2,4,6-tri-tert-butylphenyl (supermesityl or Mes*). ${ }^{18}$ The use of the bulky Mes* protecting group directly at the P-atom is possible in $C, C-\mathrm{A}_{2} \mathrm{PAs} 4$, which were thus our first synthetic targets. In accordance with the preparation of $C$-monoacetylenic phosphalkenes, ${ }^{17 a}$ a convenient synthetic approach towards Mes*-protected $C, C-\mathrm{A}_{2} \mathrm{PAs}$ might be a twofold Pd-catalyzed alkynylation of the C,C-dibromophosphaalkene 6 as a starting material. ${ }^{19}$ Such an approach based on the corresponding dibromoalkenes is commonly used for the preparation of all-carbon DEE 1 and TEE 2 building blocks (Scheme 1, route a) ${ }^{1,3}$ However, exposure of the phosphorus counterpart, the C,C-dibromophosphaalkene 6, to standard reaction conditions of Pd-catalyzed cross-coupling led to the formation of phosphaalkyne 7 and polymeric decomposition products thereof, instead of the desired C,C-diacetylenic phosphaalkenes (Scheme 1, route b). ${ }^{17 a, 19 b, 20}$ The conversion of $\mathbf{6}$ into $\mathbf{7}$ has been reported in the literature as the phosphorus version of the Fritsch-Buttenberg-Wiechell (FBW) rearrangement. ${ }^{21}$

By analogy to alkene chemistry, our approach for the formation of the acetylenic phosphaalkenes $\mathbf{1 0}$ and $\mathbf{1 1}$ was based on the reactions of propargylic and allenic chlorides 8 and $\mathbf{9}$, respectively, with $\mathrm{Mes}^{*} \mathrm{PCl}_{2}$ (Scheme 1, route c). ${ }^{16,22}$ The elimination of $\mathrm{LiCl}$ and $\mathrm{HCl}$ is promoted by $\mathrm{LDA}$, and the stereochemistry of the products is predetermined by the acetylenic substituents of the starting materials. Surprisingly, the reaction of 3-chloropenta-1,4-diynes 9 gives the butadiyne-substituted phosphaalkenes $\mathbf{1 1}$, with the Mes*group cis to the butadiyne. On the contrary, $(E)$ - and $(Z)$ C,C- $\mathrm{A}_{2} \mathrm{PAs} 10$ are formed from 1-chloropenta-1,2-dien-4ynes 8 when exposed to the same reaction conditions. In both cases, the reaction proceeds through $C_{5}$ allenyllithium intermediates that, in the case of $\mathbf{9}$, are accessible from the initially formed propargyllithium species through an isomerization reaction. ${ }^{23}$ The product outcome is dependent on whether the reaction of the $C_{5}$ fragment takes place at the terminal C1-atom or at the central C3-position.

Furthermore, it was found that the propargylic chlorides 9 can be converted into the chloroallenes 8 by a silicapromoted rearrangement. ${ }^{24}$ With the aim of expanding the synthetic procedures to $C, C-\mathrm{A}_{2} \mathrm{PAs} \mathbf{1 0}$, an additional route that omits acetylenic chloroallenes was developed. This route is applicable to the synthesis of bis(trimethylsilyl)terminated $C, C-A_{2} P A$, and is based on a propargylic Grignard reagent that, in turn, is prepared from 3-bromo-1,5bis(trimethylsilyl)penta-1,4-diyne and Rieke-Mg. ${ }^{25}$ Reaction of the Grignard reagent with $\mathrm{Mes}^{*} \mathrm{PCl}_{2}$ and subsequent treatment with DABCO facilitates dehydrochlorination and the formation of the $\mathrm{P}=\mathrm{C}$ double bond. Later, we developed an alternative route for the preparation of $C, C-A_{2} P A s ~ 10$ from the Mes*-protected C,C-dibromophosphaalkene 6 through a trans-selective sulfonyl-mediated alkynylation and a subsequent Sonogashira-Hagihara cross-coupling of the intermediate monoacetylenic C-APA 12 (Scheme 1, route d). ${ }^{26}$

A structural comparison of compounds $\mathbf{1 0}$ and $\mathbf{1 1}$ revealed that, for most of the acetylenic phosphaalkenes, a characteristic feature is the coplanarity of the $\mathrm{P}=\mathrm{C}$ and $\mathrm{C} \equiv \mathrm{C}$ 
a)

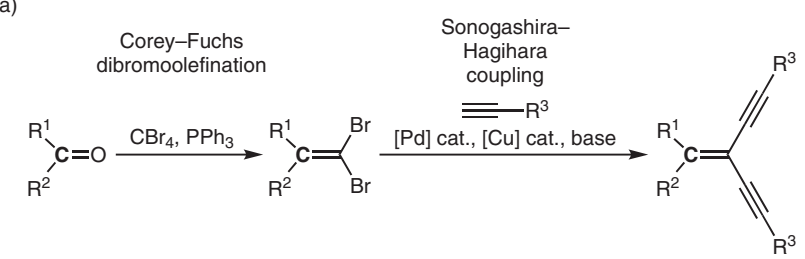
$R^{1}, R^{2}=H$, aryl, hetaryl, alkyl, ethynyl $\quad R^{3}=$ silyl, aryl $\quad 1$ or 2

b)

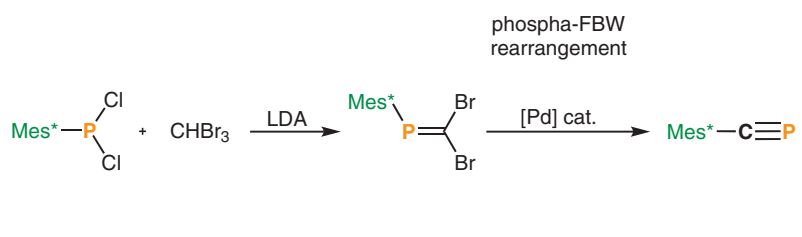
6 7

d)

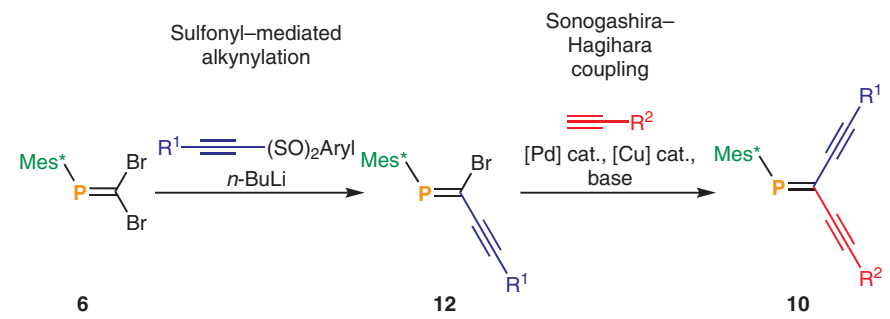

$\mathrm{R}^{1}=\mathrm{Ph}, 4-\mathrm{Br}^{-} \mathrm{C}_{6} \mathrm{H}_{4}, \mathrm{Fc}$

$\mathrm{R}^{1}=\mathrm{Ph}, \mathrm{R}^{2}=\mathrm{Fc}$ $R^{1}=F c, R^{2}=P h$

Scheme 1 Synthetic approaches to oligoenynes and C,C-diacetylenic phosphaalkenes: (a) Synthesis of gem-diethynylethenes or tri- and tetraethynylethenes from aldehydes and ketones by Corey-Fuchs dibromoolefinations and subsequent Sonogashira-Hagihara couplings. (b) Formation of C,C-dibromophosphaalkene and transition-metal-mediated phospha-FBW rearrangement to phosphaalkynes. (c) Synthesis of acetylenic phosphaalkenes from allenic or propargylic chlorides through condensation with $\mathrm{Mes}^{*} \mathrm{PCl}_{2}$. (d) Synthesis of C,C-diacetylenic phosphaalkenes from a C,C-dibromophosphaalkene through sulfonyl-mediated alkynylation and subsequent Sonogashira-Hagihara coupling.

units, which results in a substantial degree of $\pi$-conjugation, whereas the Mes*-protecting group is almost perpendicular to the extended $\pi$-system, as expected.

By analogy to the classical carbon-based gem-DEE 1 and TEE 2 units, C,C-A 2 PAs 10 and acetylenic phosphaalkenes $\mathbf{1 1}$ are appealing building blocks for the construction of more elaborate oligomeric and cyclic architectures. ${ }^{27}$ The acetylenic moieties at the C-termini of the phosphaalkenes offer a unique opportunity for extending the conjugated system with exocyclic $\mathrm{P}=\mathrm{C}$ units linked through acetylene bridges. The phosphaalkene units in the backbone have a stabilizing effect on the lowest unoccupied molecular orbitals (LUMOs) and lead to a lowering of their energy levels, whereas those of the highest occupied molecular orbitals (HOMOs) stay largely unaffected. ${ }^{27 c, 28}$ On the basis of these considerations, we prepared a number of extended molecular systems by using compounds 10a-c and $\mathbf{1 1}\left(\mathrm{R}^{1}=\mathrm{TMS} ; \mathrm{R}^{2}=\mathrm{Ph}\right)$ as modular units. Some selected systems are represented in Figure 4 in comparison with their all-carbon-based counterparts. In particular, the bis(trimethylsilyl)-terminated $C, C-\mathrm{A}_{2} \mathrm{PA} \mathbf{1 0 a}$ and the nonsymmetrically substituted $\mathbf{1 0 b}\left(\mathrm{R}^{1}\right.$ $\left.=\mathrm{Ph} ; \mathrm{R}^{2}=\mathrm{TMS}\right)$ and $10 \mathrm{c}\left(\mathrm{R}^{1}=\mathrm{TMS} ; \mathrm{R}^{2}=\mathrm{Ph}\right)$ are important starting materials because they permit the facile removal of the TMS groups under mild conditions.
Treatment of 10a with $\mathrm{CuCl}$ gives direct access to the copper(I) acetylide that is selectively formed at the acetylene moiety trans to the Mes* group, because the cis(trimethylsilyl)acetylene is sterically more protected. Conveniently, the cis-(trimethylsilyl)acetylene can be deprotected at higher temperatures and with longer reaction times. This differentiation of the acetylene termini in 10a-c is essential for the controlled synthesis of the title compounds in Figure 4. For example, compounds 16a-c were prepared by in situ generation of the intermediate copper(I) acetylides and subsequent Pd-catalyzed oxidative acetylene homo- and heterocouplings of 10a-c. Interestingly, both steps proceed without isomerization across the $\mathrm{P}=\mathrm{C}$ double bond. Compounds 16b (E-isomer) and 19a were prepared from 10b and $11\left(R^{1}=P h ; R^{2}=T M S\right)$, respectively, in a onepot deprotection-homocoupling protocol by using $\mathrm{K}_{2} \mathrm{CO}_{3}$ and the classical Eglington conditions $\left[\mathrm{Cu}(\mathrm{OAc})_{2}\right.$ in $\mathrm{MeOH}$ and pyridine]. In situ desilylation and subsequent Pd-catalyzed Sonogashira-Hagihara cross-coupling reactions on 10a and $\mathbf{1 1}\left(\mathrm{R}^{1}=\right.$ TMS; $\left.\mathrm{R}^{2}=\mathrm{Ph}\right)$ could be used to afford various aryl-terminated acetylenic phosphaalkenes and the phenylene-bridged dimeric $C, C-\mathrm{A}_{2} \mathrm{PA} 18 .{ }^{27 \mathrm{c}}$ The possibility of selective Pd-catalyzed cross-couplings on the C,C- $\mathrm{A}_{2} \mathrm{PA}$ building blocks allowed an enlargement of the acetylenic framework to the hexayne $\mathbf{1 7},{ }^{27 \mathrm{~b}}$ which is a heavier conge- 


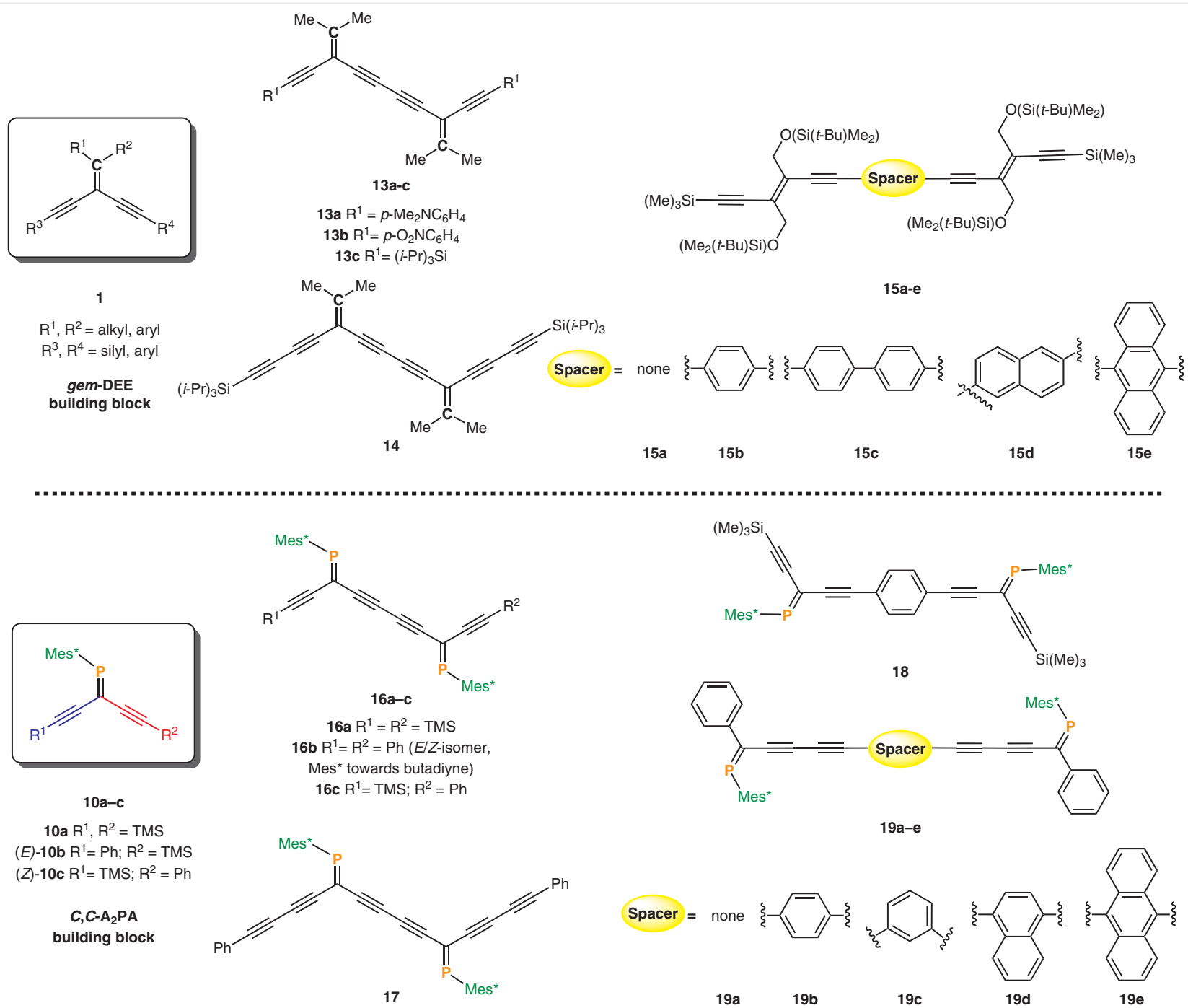

Figure 4 Selected examples of all-carbon conjugated oligoenynes (top) and oligoacetylenes with incorporated phosphaalkene units (bottom)

ner of the all-carbon-based reference system $14 .{ }^{29}$ In a separate study, monomer $\mathbf{1 1}\left(\mathrm{R}^{1}=\mathrm{TMS} ; \mathrm{R}^{2}=\mathrm{Ph}\right)$ was used with diiodoarenes as coupling partners to prepare a series of $\mathrm{P}=\mathrm{C}$-terminated acetylenic arenes $\mathbf{1 9 b}-\mathbf{e} .^{27 a} \mathrm{~A}$ structural investigation of this series showed that for the linear acetylenic compound 19a (no spacer), the entire APA backbone is coplanar, which results in a high degree of communication between the phosphaalkene units. For the dimers with a spacer unit, the best electronic communication between the two APA moieties is achieved for the bridge with the most quinoid character. This is in agreement with all-carbon-based reference compounds 15a-e, in which two trans-1,2-diethynyl-ethenes are interconnected by the same bridging aromatic units. ${ }^{1,30}$ Furthermore, the effects of the heavy alkene on the optical and electrochemical properties of the APAs (Figure 4) were investigated. As expected, substitution of $\mathrm{C}=\mathrm{C}$ units by $\mathrm{P}=\mathrm{C}$ leads to a decrease in the HOMO-LUMO gaps of the entire $\pi$-conjugates, with the phosphorus centers being integral parts. UV/Vis spectroscopy revealed some remarkable red shifts of the longest-wavelengths absorption maxima compared with those of all-carbon-based reference compounds. This effect is already pronounced in a comparison of $C, C$-APAs $10 \mathbf{a}-\mathbf{c}$ with the similarly substituted diethynylethenes gem-DEE $\mathbf{1},{ }^{31}$ and it becomes even more apparent for extended dimeric and oligomeric systems. For instance, compounds 16a-c exhibit a bathochromic shift of about $100 \mathrm{~nm}$ in comparison to the all-carbon-based $\pi$-framework 13a-c. ${ }^{29,32}$ Moreover, electrochemical data also confirm the decreased HOMO-LUMO gaps in the APA systems.

Despite these encouraging first results, further explorations of the APA systems became limited by a lack of suitable synthetic approaches and by difficulties in the preparation of new monomeric building blocks. Our aim was to 
further explore the influence of different conjugation topologies, particularly trans and cis linear conjugations, ${ }^{33}$ on the physicochemical properties of the corresponding APA systems. This quest required the preparation of $P, C$-diacetylenic $(E)-\mathbf{3},(Z)-\mathbf{3}$, and peracetylenic phosphaalkenes 5 (Figure 3). All the developed synthetic approaches to $C, C-\mathrm{A}_{2} \mathrm{PAs}$ shown in Scheme 1 use the bulky Mes* substituent to stabilize the phosphorus center and, consequently, could not be applied for the preparation of $P, C-A_{2}$ PAs 3 or $P, C, C-A_{3}$ PAs 5. The question of how to stabilize the reactive $P=C$ unit in absence of the bulky Mes* substituent then arose. One possibility might be the coordination of a metal fragment, such as $\mathrm{W}(\mathrm{CO})_{5}$, to the phosphorus atom. Therefore, two different assembly strategies were tested: (a) the formation of a $\mathrm{P}=\mathrm{C}$ double bond between $\mathrm{W}(\mathrm{CO})_{5}$-containing $P$-acetylenic and $C$-acetylenic precursors, and (b) the introduction of acetylenes at appropriately functionalized $\mathrm{P}=\mathrm{C}$ precursors with $\mathrm{W}(\mathrm{CO})_{5}$ fragments. Unfortunately, neither of these strategies was blessed with success.

At this point, the use of phosphorus olefination methods to convert carbonyl compounds into $\mathrm{P}=\mathrm{C}$ double bonds as the central reaction towards APAs seemed to be a good idea. This prompted us to move into the phosphorus versions of the Wittig and the Horner-Wadsworth-Emmons (HWE) reactions (Scheme 2). By the way, the existence of phosphorus versions of these two classic organic reactions is another example of the analogy between phosphorus and carbon. ${ }^{8,11 b, e, 34}$

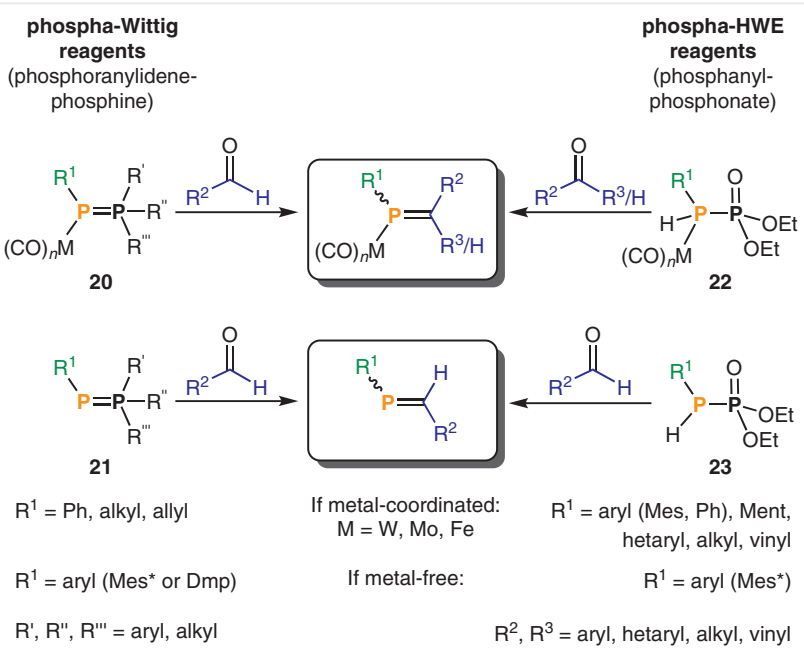

Scheme 2 Phospha-Wittig (left) and phospha-Horner-WadsworthEmmons (right) approaches for the synthesis of phosphaalkenes from aldehydes or ketones. Metal-coordinated (top) and metal-free (bottom) variations.

In the phospha-Wittig variant, phosphoranylidenephosphine (phospha-ylide) reagents $\mathbf{2 0}$ or $\mathbf{2 1}$ react with aldehydes (but not ketones) to form the corresponding phosphaalkenes. Initially, metal-coordinated (W, Mo, Cr, or Fe) reagents $\mathbf{2 0}$ were developed to stabilize both the starting material and the final products (Scheme 2, top left). ${ }^{35}$ The assistance of transition metals and complexation makes this approach general for a variety of substrates, irrespective of steric demands. Later on, the corresponding metalfree phospha-Wittig reagents $\mathbf{2 1}$ were prepared and successfully used in the synthesis of stable phosphaalkenes (Scheme 2, bottom left). ${ }^{36}$ In the case of the phospha-HWE variation, the reagents are phosphanylphosphonates $\mathbf{2 2}$ and 23 that contain an $(\mathrm{RO})_{2} \mathrm{P}=\mathrm{O}$ unit and are generally more reactive, thus allowing the use of aldehydes or ketones as substrates (Scheme 2, right-hand side).

\section{The Phospha-Horner-Wadsworth- Emmons Approach to Phosphaalkenes}

\subsection{Metal-Coordinated Phosphanylphosphonates}

The chemistry of phospha-Wittig and phospha-HWE reagents was pioneered by the group of François Mathey in the late $1980 \mathrm{~s} .{ }^{37}$ Initially, the first phosphanylphosphonate reagent 22 was prepared from the (phosphirane)pentacarbonyltungsten complex $\mathbf{2 4}$ in a ring-opening reaction upon nucleophilic attack by $\mathrm{NaP}(\mathrm{O})(\mathrm{OEt})_{2}$ (Scheme 3 ). The reagent $\mathbf{2 2}$ is generated via intermediate $\mathbf{2 5}$, which rapidly decomposes to give styrene and the anionic equivalent $\mathbf{2 6}$, which undergoes protonation. ${ }^{37 a, 38}$

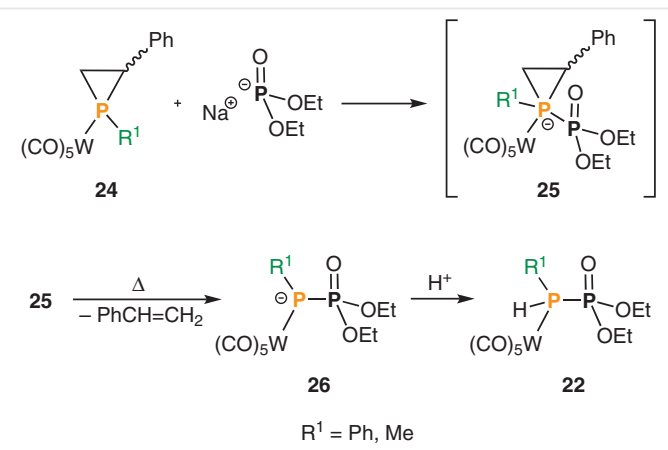

Scheme 3 Synthesis of the first W(CO) ${ }_{5}$-stabilized phosphanylphosphonate (phospha-HWE reagent) ${ }^{38}$

Further synthetic protocols were later devised by Mathey and co-workers in the early 1990s (Scheme 4, routes a-c). ${ }^{11 b, 37 b, 39}$ These routes involve precursors that are more readily available and they allow variations of the metal centers and the substituent $\mathrm{R}^{1}$ on phosphorus. Typical metals for the stabilization of $\mathbf{2 2}$ are $\mathrm{W}$, Mo, and Fe, which can be introduced from $\mathrm{M}(\mathrm{CO})_{5}(\mathrm{CN})$ for $\mathrm{W}$ and Mo or from $\mathrm{M}_{2}(\mathrm{CO})_{9}$ for Fe. Nevertheless, routes a and $\mathrm{b}$ can be synthetically challenging because they involve a twofold lithiation of the primary phosphine $\mathbf{2 9}$ and because the use of LDA can lead to side reactions from the addition of $\left[(i-\mathrm{Pr})_{2} \mathrm{~N}\right]^{-}$to the $\mathrm{ClP}(\mathrm{O})(\mathrm{OEt})_{2}$ reagent. Protocol $\mathrm{c}$, which starts with a 


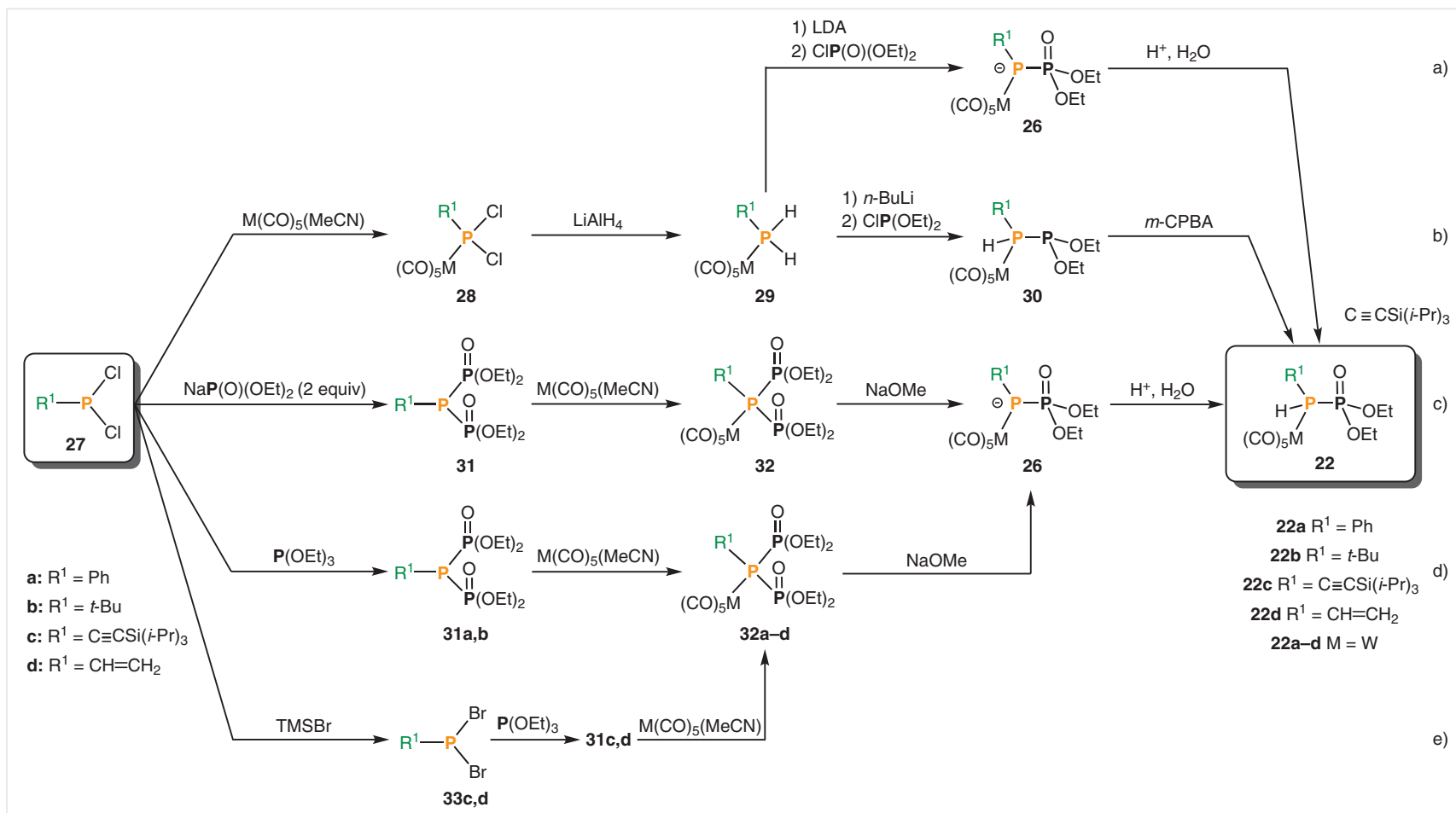

Scheme 4 Synthetic routes towards metal-coordinated phosphanylphosphonate reagents. (a) $R^{1}=P h, t-B u ; M=W, M o^{37 b}$ (b) $R^{1}=M e s$, menthyl; $M=W^{39,41}$ (c) $R^{1}=P h ; M=W, M o, F e, R^{1}=M e ; M=W, M o, R^{1}=t-B u ; M=W, F e ; R^{1}=C H=C H P h, C H=C H O B u, 2$-thienyl; $M=W^{39}(d) R^{1}=P h, t-B u ;$ $M=W^{40}(e) R^{1}=T I P S, C H=C_{2} ; M=W^{40}$

phospha-Michaelis-Becker reaction, is synthetically the most reliable, but requires precise control of added sodium for the preparation of the $\mathrm{NaP}(\mathrm{O})(\mathrm{OEt})_{2}$ reagent.

The use of Mathey's phosphanylphosphonates 22 for the formation of phosphaalkenes from carbonyl compounds (Scheme 2) had lain dormant for almost three decades until 2012, when we applied this method to the preparation of phosphaalkenes in $\pi$-conjugation with other unsaturated organic groups. ${ }^{40}$

Facing some synthetic difficulties with routes a-c in Scheme 4, we were encouraged to develop alternative approaches that permit the preparation of phospha-HWE reagents on a multigram scale (Scheme 4 , routes d-e) ${ }^{40}$

In this context, the phospha-Michaelis-Arbuzov reaction seemed to be a viable alternative for converting $P, P$-dichlorophosphines 27a,b into the corresponding bis(phosphoryl)phosphines 31a,b. Subsequent complexation with $\mathrm{W}(\mathrm{CO})_{5}(\mathrm{MeCN})$ and selective cleavage of one phosphorylphosphine bond with sodium methoxide yields the anionic forms $\mathbf{2 6 a}, \mathbf{b}$, which can be protonated to give the phosphaHWE reagents 22a,b (Scheme 4, route d). In fact, 22a could be prepared from 27a on a 15 gram scale with a total yield of over $62 \%$ over three steps. ${ }^{40}$

However, the formation of the intermediates $\mathbf{3 1}$ is strongly depending on the nature of the substituent $R^{1}$. Whereas in the case of $\mathbf{2 7 a}, \mathbf{b}\left(\mathrm{R}^{1}=\mathrm{Ph}, t-\mathrm{Bu}\right)$, the desired bis(phosphoryl)phosphines 31a,b are obtained in good yields, the reactions of dichlorophosphines $27 \mathrm{c}, \mathbf{d}\left(\mathrm{R}^{1}=\right.$ TIPS, $\mathrm{C}=\mathrm{CH}_{2}$ ) with triethyl phosphite are sluggish and result in mixtures of ethylchlorophosphites with varying numbers of chloro and ethoxy groups. To circumvent these problems, 27c,d were converted into the corresponding $P, P$-dibromophosphines 33c,d by using trimethylsilyl bromide. The subsequent phospha-Michaelis-Arbuzov reaction of the dibromides with triethyl phosphite smoothly affords the bis(phosphoryl)phosphines 31c,d (Scheme 4, route e), which are further transformed into the anions $\mathbf{2 6 c}$,d. As mentioned above, the quenching of $\mathbf{2 6 a}, \mathbf{b}$ gives the desired reagents $\mathbf{2 2 a} \mathbf{a}, \mathbf{b}$, whereas in the case of the appended $\pi$-system-containing $\mathbf{2 6 c}, \mathbf{d}$, a more complicated picture emerges. As a matter of fact, compounds $\mathbf{2 2 c}$, $\mathbf{d}$ are elusive and follow-up products are formed (Scheme 5). For example, the TIPS-ethynyl-substituted 26c gives $\mathbf{4 2}$ after treatment with aqueous $\mathrm{NH}_{4} \mathrm{Cl}$, whereas $\mathbf{2 6 d}$ gives another major product 43 by dimerization of two $26 \mathbf{d}$ units through the vinyl groups. This dimerization can, however, be avoided by performing the quenching under strongly acidic conditions. ${ }^{40}$

In general, anions $\mathbf{2 6}$ can be formed from the corresponding stable phosphanylphosphonate complexes 22 upon treatment with a base such as n-BuLi, 1,4-diazabicyclo[2.2.2]-octane (DBO), or lithium diisopropylamide (LDA). These in situ-generated anions $\mathbf{2 6}$ react with aldehydes or 


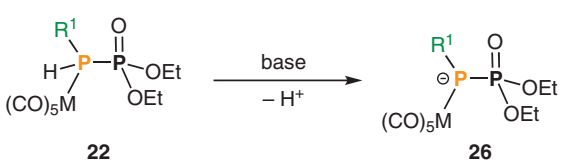

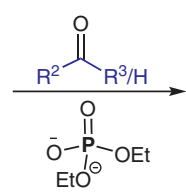

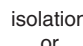

trapping<smiles>[R]C(P)=P([R])([13CH3])[18OH]</smiles>
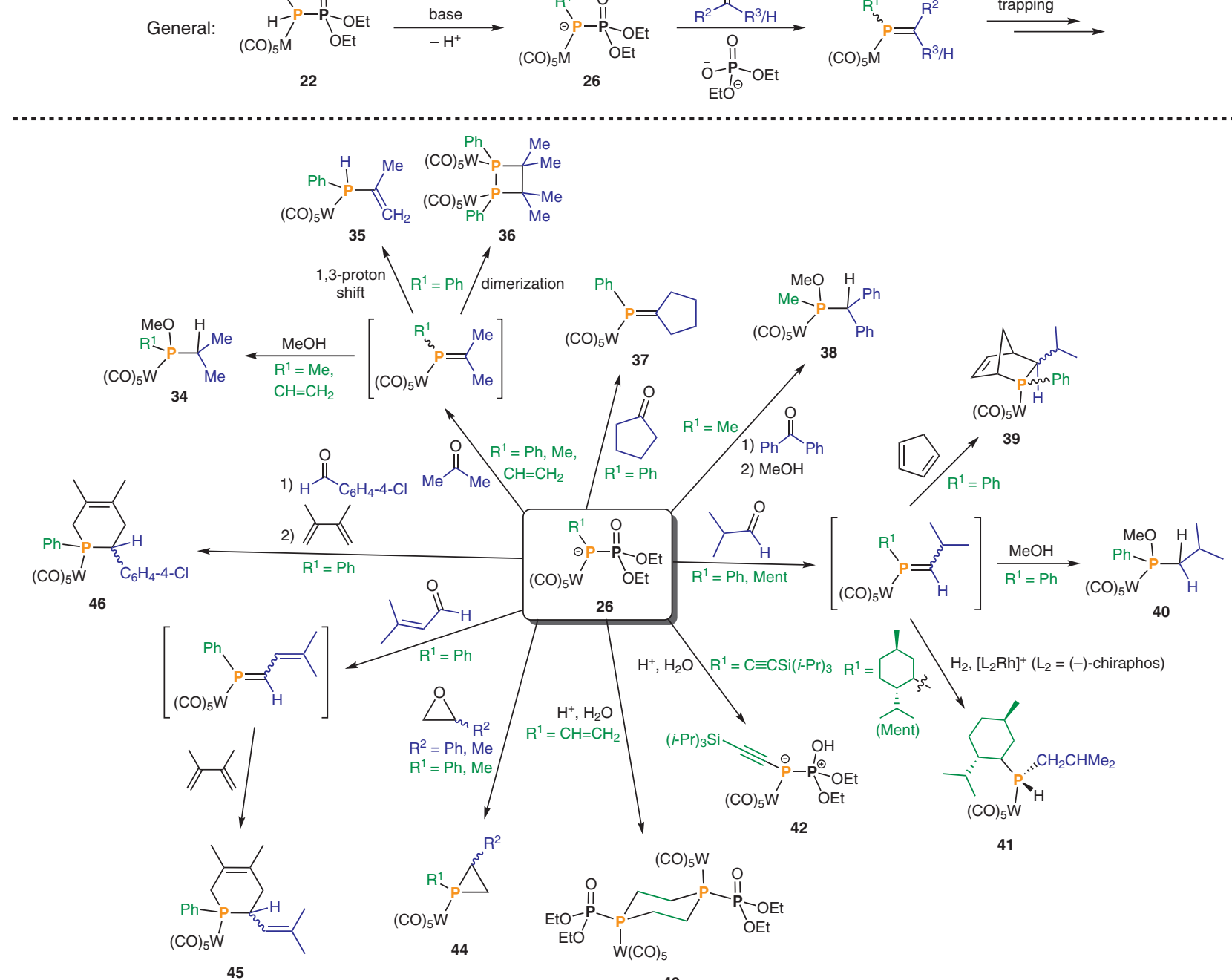

43

Scheme 5 Selected examples of the broad scope of reactivity of metal-coordinated phosphanylphosphonates

ketones to yield the corresponding phosphaalkene complexes (Scheme 5, top). Depending on the stability of these complexes, they can be either isolated (e.g., compound 37) or trapped with various reagents such as methanol or buta1,3-dienes to give phosphinite complexes (e.g., $\mathbf{3 4}, \mathbf{3 8}$, and 40) or tetrahydrophosphinine complexes (e.g., 45 and 46), respectively. ${ }^{11 b, 34,37,40-42}$ The stability of the phosphaalkene complexes is determined by the steric nature of the substituent $\mathrm{R}^{1}$ on the anionic P-center and the substituents of the carbonyl compounds, by the inherent stability of the metal complex formed, and by the nature of the trapping agent. Additionally, steric factors also play an important role in the stereochemistry of the final products. ${ }^{34}$ Numerous products that contain $\mathrm{P}-\mathrm{C}$ single bonds, $\mathrm{P}=\mathrm{C}$ double bonds, or $\mathrm{P}-\mathrm{C}$ heterocycles have been prepared from $\mathbf{2 2}$ as a starting material (Scheme 5), illustrating its broad reactivity of what seemingly is a simple phospha-version of the HWE reac- tion. For example, the reaction of complex $\mathbf{2 6 a}$ with acetone yields the corresponding phosphaalkene after several minutes. Upon prolonged reaction times, the intermediate phosphaalkene is consumed, and secondary vinylphosphane 35 and 1,2-diphosphitane 36 are formed by a 1,3proton shift or a head-to-head dimerization, respectively. ${ }^{40}$ If 26a is treated with an $\alpha, \beta$-unsaturated aldehyde, the corresponding 1 -phosphadiene can be trapped by conjugated dienes such as 2,3-dimethylbuta-1,3-diene, which gives the $[2+4]$-cycloadduct $45 .{ }^{37 \mathrm{c}}$

Interestingly, anions $\mathbf{2 6}$ can react with oxiranes to form phosphirane complexes $\mathbf{4 4}$, thus demonstrating reactivity with oxygen-containing substrates other than carbonyl compounds. ${ }^{11 \mathrm{~b}}$ Furthermore, Mathey and co-workers have shown that the phospha-HWE reaction can also be used for the preparation of prochiral phosphaalkene complexes with $\mathrm{L}$-menthyl as the $\mathrm{R}^{1}$ group. These complexes can undergo 
catalytic hydrogenation to yield optically active phosphines 41, which underlines once more the wide variety and broad scope of the phospha-HWE reaction. ${ }^{41}$

\subsection{Mechanism of the Phospha-Horner- Wadsworth-Emmons Reaction}

In the classic Horner-Wadsworth-Emmons (HWE) carbonyl olefination, the crucial step for the formation of the $\mathrm{C}=\mathrm{C}$ double bond from the intermediate oxaphosphetane is a simultaneous $\mathrm{P}-\mathrm{C}$ and $\mathrm{C}-\mathrm{O}$ bond cleavage. ${ }^{43}$ The mechanism for the phosphorus analogue of the HWE reaction is more complex and proceeds in a stepwise fashion. From a study of the reaction between the phosphanylphosphonates 22a,b and two ketene substrates $4 \mathbf{4 a}, \mathbf{b}$, we proposed the detailed mechanism depicted in Scheme $6 .^{44}$ The elucidation of the mechanism is based on the isolated reaction intermediates 48a,b and 49a-d which were characterized spectroscopically and crystallographically.

The reaction is initiated by the addition of a stoichiometric amount of an organic base (e.g., DBU) to compounds $\mathbf{2 2 a}, \mathbf{b}$ followed by the addition of ketene $\mathbf{4 7} \mathbf{a}$ or $\mathbf{4 7 b}$. After 30 minutes of reaction time and aqueous workup, to our surprise we observed the formation of phosphinophosphates 49a-d instead of the expected phosphaallenes. However, when the reaction times were extended to a couple of days, the corresponding phosphaallenes $\mathbf{5 0 a}-\mathbf{d}$ were formed. By using an organolithium base, such as LDA or $t$ $\mathrm{BuOLi}$, the reaction times could be significantly reduced, and the phosphaallenes $\mathbf{5 0 a - d}$ were formed within one hour without the detection of any reaction intermediates. When the reaction was conducted with substoichiometric amounts of base, intermediates $48 \mathbf{a}$,b were obtained from the reaction of 22a,b with $47 \mathbf{a}$. Both intermediates 48 and 49 could be induced to reenter the reaction, simply by addition of a base. Whereas the transformation of 48 into 49 occurred in minutes, the final elimination to afford $\mathbf{5 0}$ proceeded on a similar timescale to those required in the onepot reaction. Thus, the rate-limiting step is the elimination of the phosphate group with the concomitant formation of the phosphaallene. The phosphaallene products, however, are highly sensitive toward moisture and they have low stability, so that trapping with either water or methanol is required.

On the basis of the isolated intermediates $\mathbf{4 8}$ and $\mathbf{4 9}$, we proposed a mechanistic sequence, starting with the nucleophilic attack of the PIII center of the phosphanylphosphonate at the ketene carbon. After the formation of intermediate $\mathbf{A}$, a subsequent intramolecular ring closure gives the oxadiphosphetane $\mathbf{B}$, which is analogous to the oxaphosphetane in the HWE reaction. The next step differs from that in the mechanism of the HWE reaction, and intermediate $\mathbf{B}$ does not undergo a single-step phosphate elimination. Instead, the P-P single bond is cleaved, yielding intermediate $\mathbf{C}$. This behavior is due to the rather weak $\mathrm{P}-\mathrm{P}$ bond and the stronger $\mathrm{sp}^{2}-\mathrm{C}-\mathrm{O}$ bond in $\mathbf{B}$ in comparison to the corresponding $\mathrm{C}-\mathrm{P}$ and $\mathrm{sp}^{3}-\mathrm{C}-\mathrm{O}$ bonds in oxaphosphetanes. The resulting intermediate $\mathbf{C}$, which contains a phosphate group and a delocalized negative charge, is best described as a phosphaallyl anion. Subsequent [2,3]-sigmatropic rearrangement gives intermediate $\mathbf{D}$. The driving force in this step is the creation of a new $\mathrm{P}-\mathrm{O}$ bond and a relatively stable vinyl carbanion. Finally, E2 elimination as the rate-limiting step establishes the 1-phosphaallenes $\mathbf{5 0 a - d}$ and the phosphate byproduct.

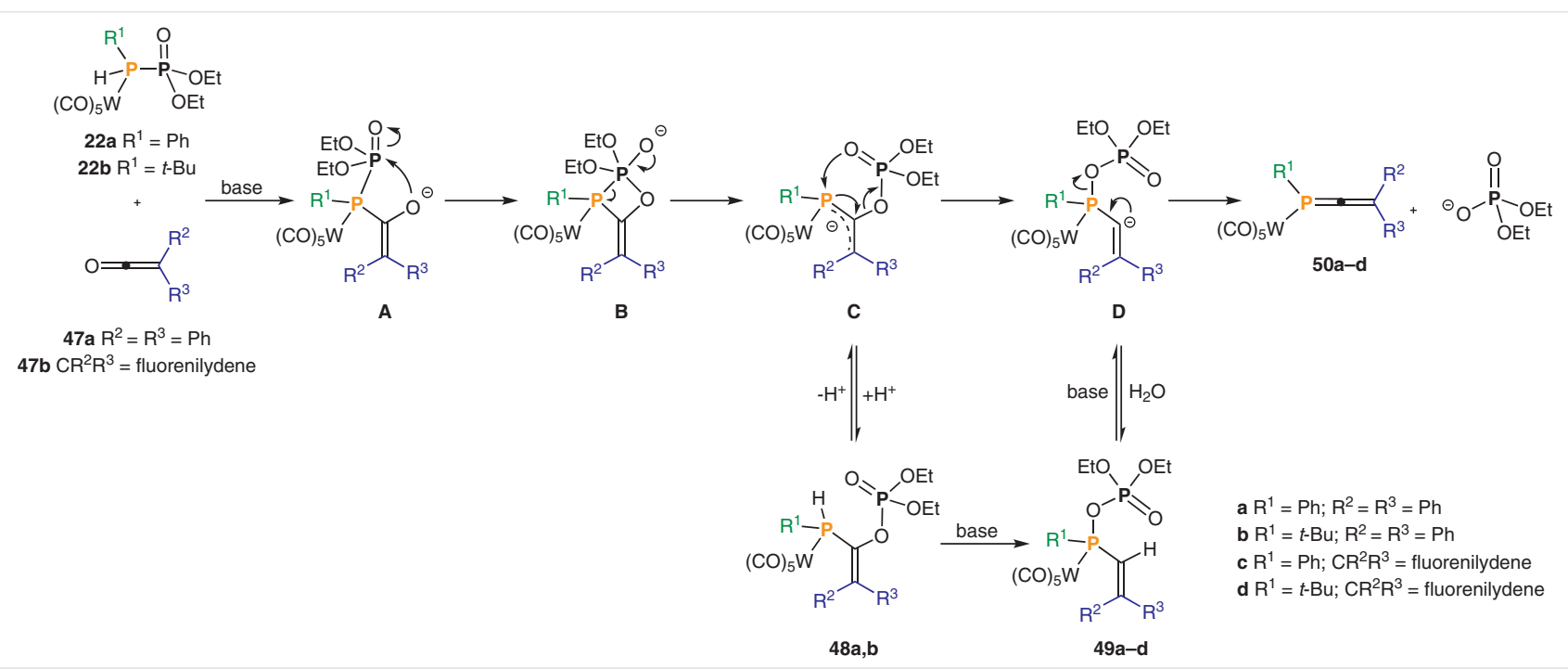

Scheme 6 Proposed mechanism of the phospha-Horner-Wadsworth-Emmons reaction ${ }^{44}$ 


\subsection{The First Metal-Free Phosphanylphosphonate and Its Reactivity with Aldehydes}

Until 2016, the usual method for the stabilization of phosphanylphosphonates was by metal-coordination to the lone pair of the PIII center. In this way, the phospha-HWE reaction afforded metal complexes as products (Scheme 5). However, the presence of such metal fragments limits subsequent chemistry, since they occupy the lone pair and impair further postsynthetic modifications at the PIII center, such as tuning of the optical properties through oxidation. These drawbacks, as well as the need for laborious, if not impossible, removal of the $\mathrm{M}(\mathrm{CO})_{5}$ fragments from the products, motivated us to design a stable and isolable phosphanylphosphonate reagent $\mathbf{2 3}$ that is free of transition metals. As already introduced in Section 2, an alternative method to metal complexation is the use of a bulky substituent such as Mes* to provide sufficient kinetic stabilization of the low-valent phosphorus center. Such a strategy permits the isolation of the reagent and the corresponding phosphaalkene products. For instance, it was applied by Protasiewicz and co-workers for the preparation of the first metal-free phosphoranylidenephosphines 21 as true phosphorus analogues of the original Wittig-type ylide (Scheme 2). ${ }^{36,45}$ In view of the successful kinetic stabilization of the phosphoranylidenephosphines, we implemented the same strategy for phosphanylphosphonates. The synthetic route towards the first metal-free phosphanylphosphonate 23a is depicted in Scheme $7 .^{46}$ We chose Mes* as the protecting group and we decided to use $\mathrm{Mes}^{*} \mathrm{PH}_{2} \mathbf{5 1}$ as a starting material because this is a very stable phosphine and can be prepared on a large scale and in high yields. ${ }^{47}$ Chlorination of $\mathrm{Mes}^{*} \mathrm{PH}_{2}$ by $\mathrm{CCl}_{4}$ and AIBN affords the monochlorinated secondary phosphine $\mathbf{5 2},{ }^{48}$ which is used for the next step without further purification. In a phospha-Michaelis-Arbuzov reaction, intermediate $\mathbf{5 2}$ undergoes a reaction with triethyl phosphite to yield the desired reagent 23a (Scheme 7). The reaction proceeds in good overall yields and can be conducted on a multigram scale.

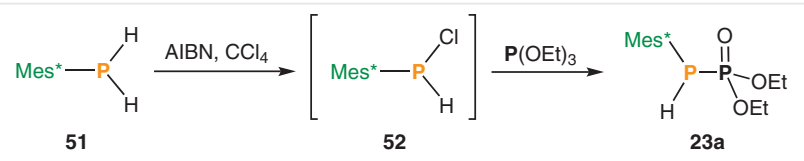

Scheme 7 Synthesis of metal-free phosphanylphosphonate reagent $\mathbf{2 3}^{\mathbf{4 6}}$

Phosphanylphosphonate 23a is remarkably stable and can be stored under an inert atmosphere at $-20{ }^{\circ} \mathrm{C}$ for several months without any observable decomposition. However, if it is exposed to moisture, water can act as a nucleophile causing slow decomposition to $\mathrm{Mes}^{*} \mathrm{P}(\mathrm{O}) \mathrm{H}_{2}$ and $\mathrm{HP}(\mathrm{O})(\mathrm{OEt})_{2}$.

In analogy to the procedure in Scheme 5, the transition metal-free phosphanylphosphonate 23a can also be applied in phospha-HWE reactions with aldehydes to afford stable phosphaalkenes (Table 1 ). This reaction proceeds under mild conditions and with a diverse range of aldehydes with aromatic, heterocyclic, aliphatic, or vinylic substituents. The corresponding substrate scope is summarized in Table 1.

Table 1 Substrate Scope for the Transition-Metal-Free Phospha-HWE Reaction $^{\mathrm{a}}$
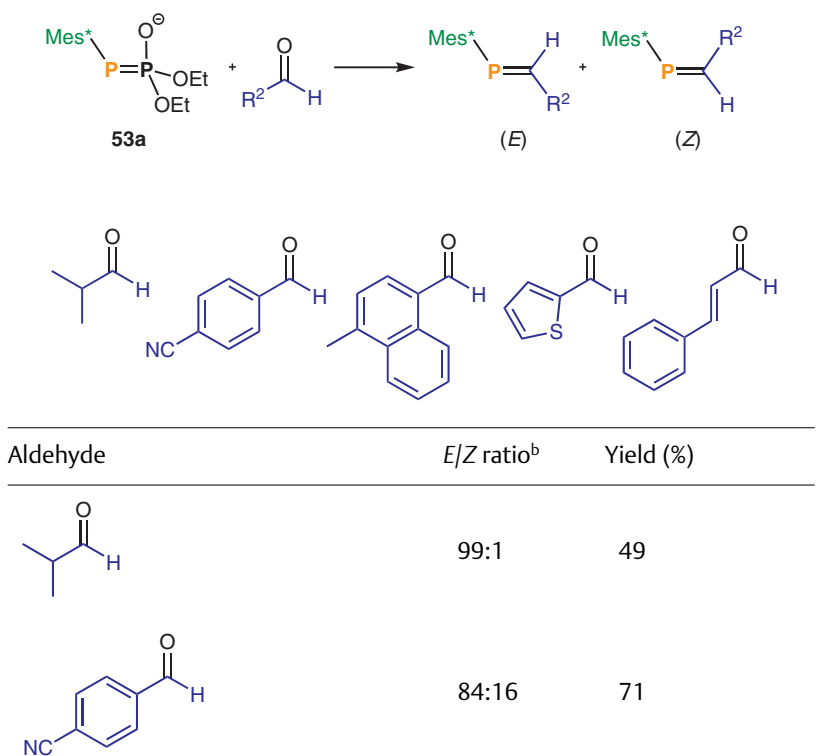<smiles>Cc1cccc2ccccc12</smiles>

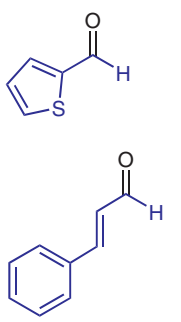

$92: 8$

a Reaction conditions: LDA (1.1 equiv) for deprotonation of 23a to 53a, aldehyde (4 equiv), $-50{ }^{\circ} \mathrm{C}$ to r.t. in THF, overnight.

${ }^{\mathrm{b}}$ Isomeric ratio before purification.

In the reaction, LDA is used to deprotonate 23a to the corresponding anionic phospha-HWE reagent 53a. From an analysis of the ${ }^{1} J_{\mathrm{P}-\mathrm{P}}$ coupling constants, it was shown that anion 53a is best described as an enolate since the ${ }^{1} J_{\mathrm{P}-\mathrm{P}}$ values are in the same range as those for phosphoranylidenephosphines. Furthermore, anionic and metal-free 53a has also significantly larger coupling constant than the corresponding W- and Mo-coordinated analogues. This phenomenon points towards a decreased bond order in the latter due to $\pi$-backbonding from the transition metal to the $\mathrm{P}=\mathrm{P}$ $\pi^{*}$ orbital. 
In general, the phospha-HWE reaction has a high $E$-selectivity, but $E / Z$-isomerization can occur during workup and purification. In particular, standard acidic silica gel column chromatography promotes $E / Z$-isomerization.

\section{Reactions with Acetylenic Ketones}

After our initial synthetic targets of acetylenic phosphaalkenes, we investigated the reactivity of phosphanylphosphonates with acetylenic ketones. ${ }^{49}$

\subsection{Metal-Coordinated Phosphanylphosphonate and Monoacetylenic Ketones}

In early studies, the metal-coordinated phosphanylphosphonate 22a was used in reactions with monoacetylenic substrates. ${ }^{49 a}$

Much to our surprise, the reaction of 22a with acetylenic ketones 54a-e does not follow the expected phosphaHWE reactivity, but proceeds by a [2+2]-cycloaddition to afford, ultimately, the 2,5-dihydro-1,2-oxaphospholes 55ae and 56a-e (Scheme 8). Depending on the substituent at the acetylene terminus, the reaction outcome can be tuned, and the desired phosphaalkene is formed if the ketone has bulky groups, such as the TIPS group in 54f. However, the phosphaalkene $\mathbf{5 7}$ exhibits a low stability and can only be isolated as its methanol addition product $\mathbf{5 8}$.

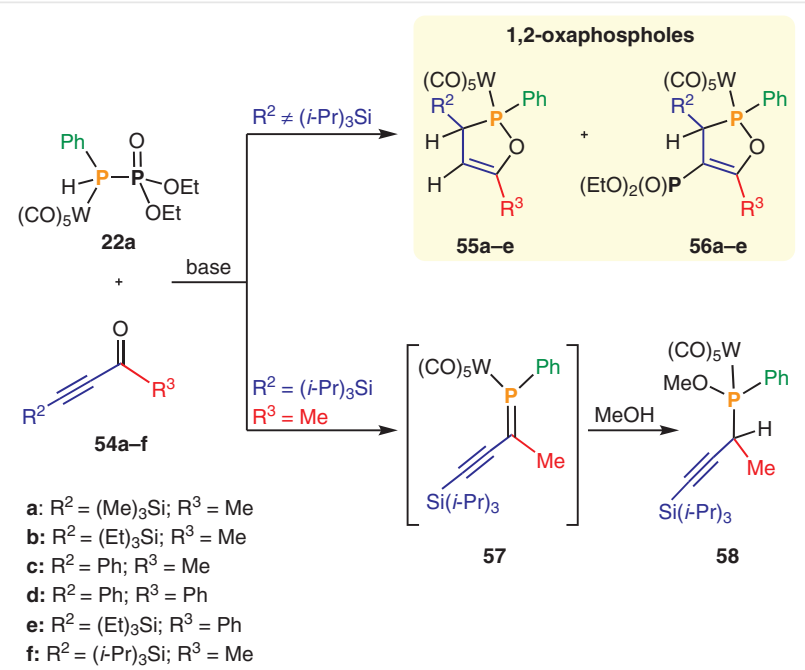

Scheme 8 Reaction of metal-coordinated phosphanylphosphonate 22a with monoacetylenic ketones
1,2-Oxaphospholes 55a-e and 56a-e differ only in the presence of a phosphate group at the $C 4$ carbon atom of the heterocycle, and the ratio between $55 \mathbf{a}-\mathbf{e}$ and $56 \mathbf{a}-\mathbf{e}$ strongly depends on the $\mathrm{pH}$ during the final quenching and workup. If the reaction is terminated by an acidic quenching (e.g. pouring onto silica gel), oxaphospholes $\mathbf{5 6 a - e}$ are formed exclusively, whereas a basic workup leads to cleavage of the phosphate group, yielding compounds 55a-e.

\subsection{Metal-Coordinated Phosphanylphosphonate and Diacetylenic Ketones}

When we introduced a second acetylene into the ketone substrate, various cascade reactions were initiated that ultimately resulted in the formation of persubstituted 1,2-oxaphospholes 62b,c with conjugated cumulene frameworks, as well as the highly-substituted, conjugated bisphospholes 63d,e. ${ }^{49 a, b}$ As depicted in Scheme 9, the outcome is entirely determined by the nature of the remote acetylene termini. Again, only substrate 59a with bulky TIPS groups gives a phosphaalkene 60 that can be trapped in a [4+2] heteroDiels-Alder reaction with 2,3-dimethylbuta-1,3-diene to form 61. For ketones 59b,c with small substituents, such as TMS or $\mathrm{SiEt}_{3}$ (TES), the products are cumulenes $\mathbf{6 2} \mathbf{b}, \mathbf{c}$. When the terminal silyl groups on the acetylenes are replaced with aromatic substituents, the reactivity of the system changes dramatically. The sole products from the reaction of 22a with bis(phenylethynyl)ketone 59d and bis(2thiophenylethynyl)ketone 59e are the ethenyl-bridged bisphospholes 63d and 63e, respectively. Compounds 63d,e are both highly interesting molecules and feature a $2 \mathrm{e}^{-} / 2 \mathrm{H}^{+}$ redox platform with chemically reversible oxidations of the two hydroxy groups. ${ }^{50}$ The oxidation to the corresponding bisphosphol-3-ones changes the conjugation topology, which induces a hypsochromic shift in the UV/Vis spectra of the compounds. However, the back reduction proceeds through deprotonated forms of $\mathbf{6 3 d}, \mathbf{e}$ as dianionic intermediates.

The formation of such highly complex products from rather simple starting materials involves a mechanism with multiple bond-breaking and bond-forming steps. Its complexity is illustrated in Scheme 10, which summarizes the various pathways to the corresponding products. 


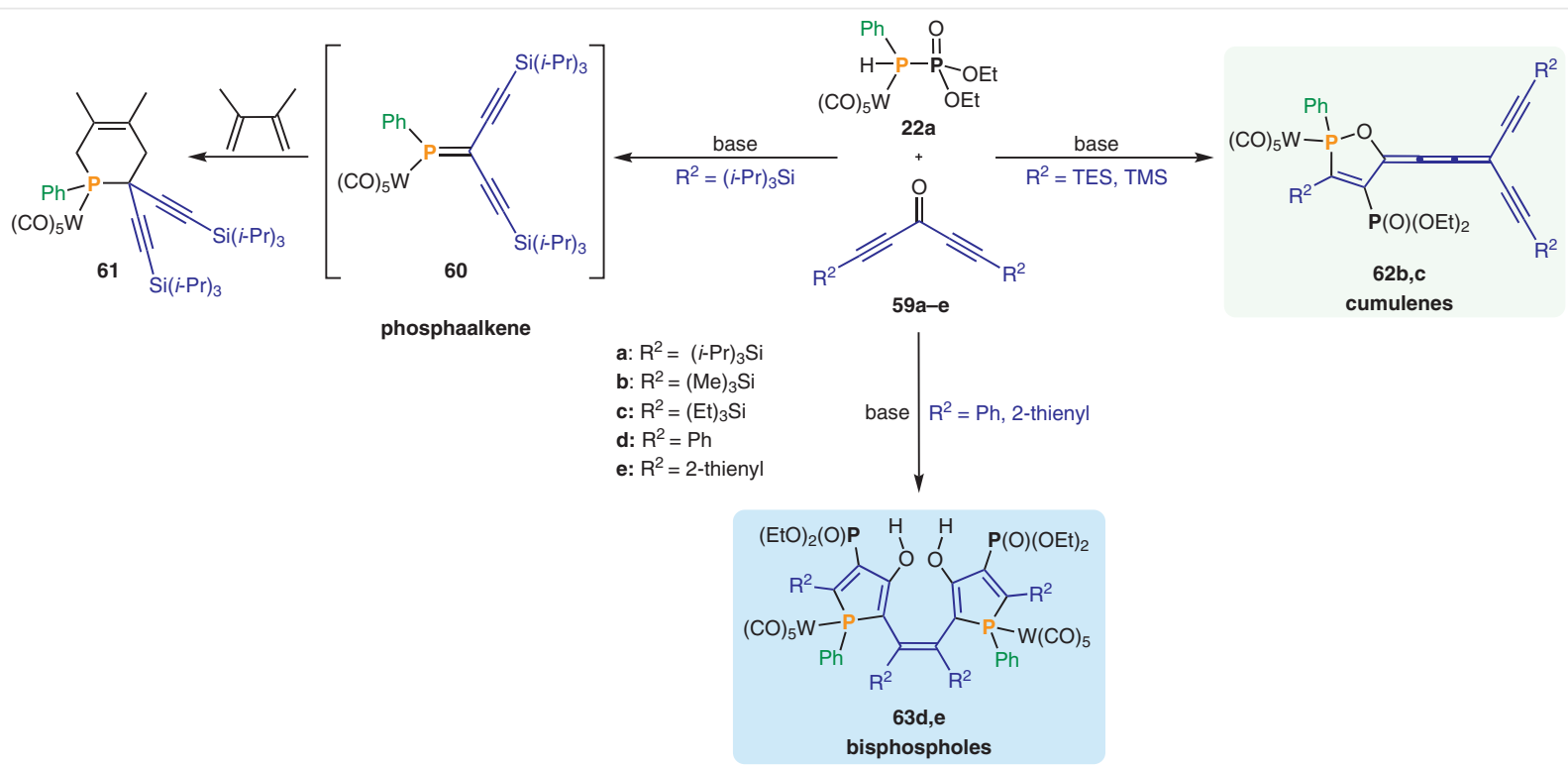

Scheme 9 Reaction of metal-coordinated phosphanylphosphonate 22a with symmetric diacetylenic ketones

For all substrates, the initial steps are identical and start with a nucleophilic attack by the PIII center of the deprotonated phosphanylphosphonate 26a at one of the acetylene termini of the ketone in a Michael fashion, followed by ring closure to yield the heterocycle E. Such reactivity has a precedent in the classic Wittig reaction with acetylene-substituted carbonyl compounds, and it can be controlled by a fine balance between electronic and steric factors. ${ }^{51}$ The Michael addition is prevented for bulky acetylene substituents, as is the case in $\mathbf{5 4 f}$ and 59a. The intermediate $\mathbf{E}$ undergoes a ring opening to form two resonance structures that carry a negative charge at either the enolate- $O$ atom $\left(\mathbf{F}_{\mathbf{A}}\right)$ or at the PIII atom $\left(\mathbf{F}_{\mathbf{B}}\right)$. These intermediates are key to the reaction's product outcome and are a formal junction point in the mechanism. In an intramolecular fashion, the PII center is nucleophilically attacked by the enolate-O atom in $\mathbf{F}_{\mathbf{A}}$ to establish the 1,2-oxaphosphole ring in $\mathbf{G}_{\mathbf{A}}$. In the case of monoacetylenic substrates, aqueous workup leads to hydrolysis of $\mathbf{G}_{\mathbf{A}}$ to form the 1,2-oxaphosphole products 55 and 56a-e. In the presence of a second acetylene unit in $\mathrm{R}^{3}$ (diacetylenic ketones $\mathbf{5 9 b}, \mathbf{c}$ with $\mathrm{SiR}_{3}$ groups), the additional resonance structures $\mathbf{G}_{\mathbf{B}}$ and $\mathbf{G}_{\mathbf{C}}$ are possible. Of these, $\mathbf{G}_{\mathbf{C}}$ has an allenyl anion in its periphery that is conveniently set up to engage in a nucleophilic attack on the carbonyl carbon of a second ketone. The adduct from this nucleophilic attack subsequently undergoes a 1,3silyl shift to give $\mathbf{H}$, in which the oxysilyl group functions as leaving group. Quenching of the reaction mixture establishes the final butatriene framework of the cumulenes $\mathbf{6 2} \mathbf{b}, \mathbf{c}$. It is interesting to note that the formation of the oxaphosphole in the first part of the reaction sequence is faster than the attack of $\mathbf{G}_{\mathbf{c}}$ on the second equivalent of the ketone.
With such a reactivity, the substitution pattern of the acetylene termini at the cumulene and the $\mathrm{C} 5$ carbon of the oxaphosphetane ring can be controlled by a stepwise addition of the corresponding ketones at different stages of this onepot reaction. Moreover, asymmetric diacetylenic ketones can also be utilized, allowing variation of substituents $R^{2}$ and $\mathrm{R}^{3}$ in the final products. ${ }^{49 a}$

In contrast to the described mechanistic sequence for silyl-terminated diacetylenic ketones, the presence of aromatic groups in 59d,e alters the reactivity dramatically. At the stage of the intermediate $\mathbf{F}$, the PII anion in the resonance structure $\mathbf{F}_{\mathbf{B}}$ can perform a 5-exo-dig attack on the carbon atom of the second acetylene unit. The resulting intermediate $\mathbf{I}_{\mathbf{A}}$ can be represented in a second resonance form $\mathbf{I}_{\mathbf{B}}$ which has an exocyclic carbon center with a certain carbene character. Dimerization of the two carbenes $\mathbf{I}_{\mathbf{B}}$ establishes a bridging double bond, and protonation affords the final bisphospholes 63d,e.

The mechanism for the formation of all heterocyclic products is supported by density functional theory calculations, which suggest that the reactivity is dictated by thermodynamic as well as kinetic aspects. It is found that the formation of the bisphospholes through carbene dimerization is significantly more exothermic for ketones with aromatic substituents on the acetylenes, whereas the cumulenic oxaphospholes are highly favored in the case of silylterminated ethynyl ketones. As described, the pathway to the latter compound includes a 1,3-silyl shift that proceeds along a smooth potential-energy surface for silyl substituents, but has a high-lying transition state if the substituents are aryl groups. ${ }^{49 a}$ 


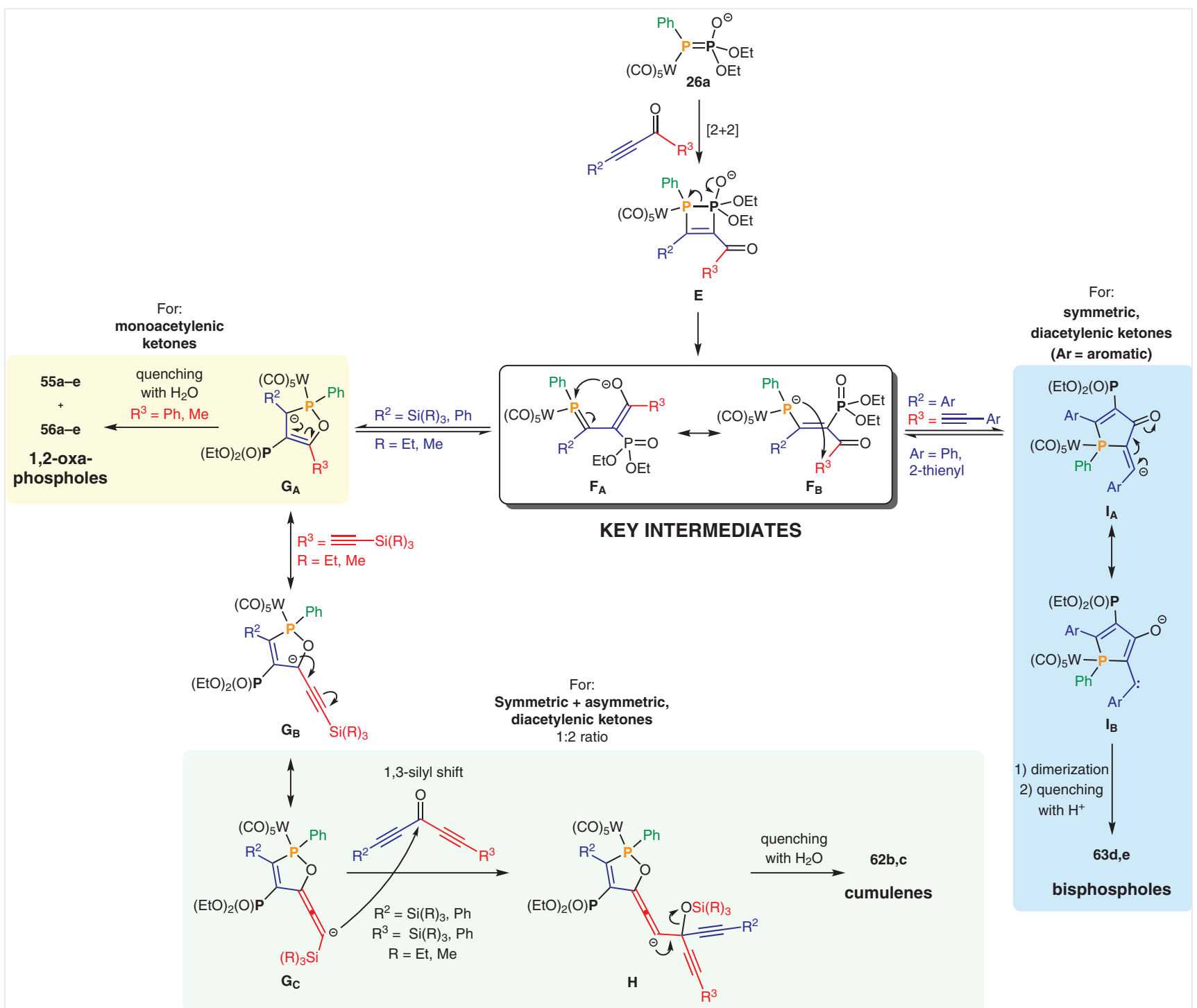

Scheme 10 Summarized mechanism for the formation of various products by the reactions of a metal-coordinated phosphanylphosphonate with acetylenic ketones ${ }^{49 a, b}$

\subsection{Metal-Free Phosphanylphosphonate and Dia- cetylenic Ketones}

Obermeier and Arkhypchuk recently explored the reactivity of the metal-free phosphanylphosphonate 23a toward symmetric diacetylenic ketones 64a-d with terminal aromatic and heterocyclic groups. ${ }^{4 c}$ In comparison with the tungsten-coordinated phosphanylphosphonate 22a, the metal-free 23a gives rise to different products. When deprotonated 23a is treated with a series of diacetylenic ketones followed by aqueous workup, phospholones 65a-d are formed (Scheme 11, part a). The product stability is dependent on the nature of the terminal substituents of the ketone and it has a substantial influence on the isolated yields. The reaction gives high yields (up to $90 \%$ ) for ketones 64a,b bearing all-carbon aromatic rings, whereas for ketones $\mathbf{6 4 c , d}$ with heterocyclic $\mathrm{R}^{2}$ groups, the yields are much lower (about 35\%).

Mechanistically, the formation of the phospholones proceeds in two steps (Scheme 11, part b). The first step, i.e. the reaction between deprotonated 23a and one of the acetylene units of the ketone is in accordance with the described mechanism for the metal-coordinated reagent 22a in Scheme 10. After ring-opening of the heterocycle $\mathbf{J}, \mathbf{a}$ phosphaalkene intermediate $\mathbf{K}_{\mathbf{A}}$ is formed (Scheme 11). Again, the intermediates can exist in several resonance forms $\mathbf{K}_{\mathrm{A}-\mathrm{C} .}$ In contrast to the case of $\mathbf{2 2 a}$ (Scheme 10), the anionic PlII center in intermediate $\mathbf{K}_{\mathbf{B}}$ does not undergo a 
nucleophilic attack on an acetylenic C-atom to form heterocycle $\mathbf{L}_{\mathbf{A}}$, which could possibly be protonated to yield the phospholones 65a-d or dimerize to yield a bisphosphole, as in case of the tungsten-protected analogue. Instead, owing to the high stability of intermediates $\mathbf{K}_{\mathbf{A}-\mathbf{c}}$, aqueous workup is required to initiate the formation of the final phospholone products. During the aqueous workup, the intermediate $\mathbf{K}_{\mathbf{A}}$ is protonated, and subsequent keto-enol tautomerization of $\mathbf{M}_{\mathbf{A}}$ yields the secondary phosphine $\mathbf{M}_{\mathbf{B}}$. In the final step, a hydrophosphination addition reaction results in formation of the phospholones 65a-d featuring an exocyclic $\mathrm{C}=\mathrm{C}$ double bond with a $Z$-configuration (Scheme 11).

The phospholones 65a-d offer interesting possibilities for postsynthetic modifications, due to the presence of trivalent phosphorus atoms and ketone functionalities in conjugation with exocyclic double bonds. Hence, the chemical behavior of compound 65a was explored in reactions with oxidants and reducing agents. It was found that both oxidation of the P-atom and conjugate reduction of the exocyclic double bond in 65a lead to substantial changes in the opto- electronic properties of the compounds, and hypsochromic shifts of the longest-wavelength absorption maximum of about 80-100 nm occurred.

\section{Metal-Free Phosphanylphosphonate as a Coupling Reagent for Aldehydes}

During our studies on metal-free phosphanylphosphonates and their reactivity toward various carbonyl compounds, a serendipitous observation attracted our attention. ${ }^{52}$ It emerged that the phospha-HWE reaction between phosphanylphosphonate 53a and 4-formylbenzonitrile resulted in the formation of an unexpected byproduct in addition to the expected phosphaalkene 66 (Scheme 12). A colorless crystalline solid that displayed an intense blue fluorescence was isolated in 5\% yield, and careful analysis revealed that this byproduct was $(E)-4,4$ '-ethene-1,2-diyldibenzonitrile.

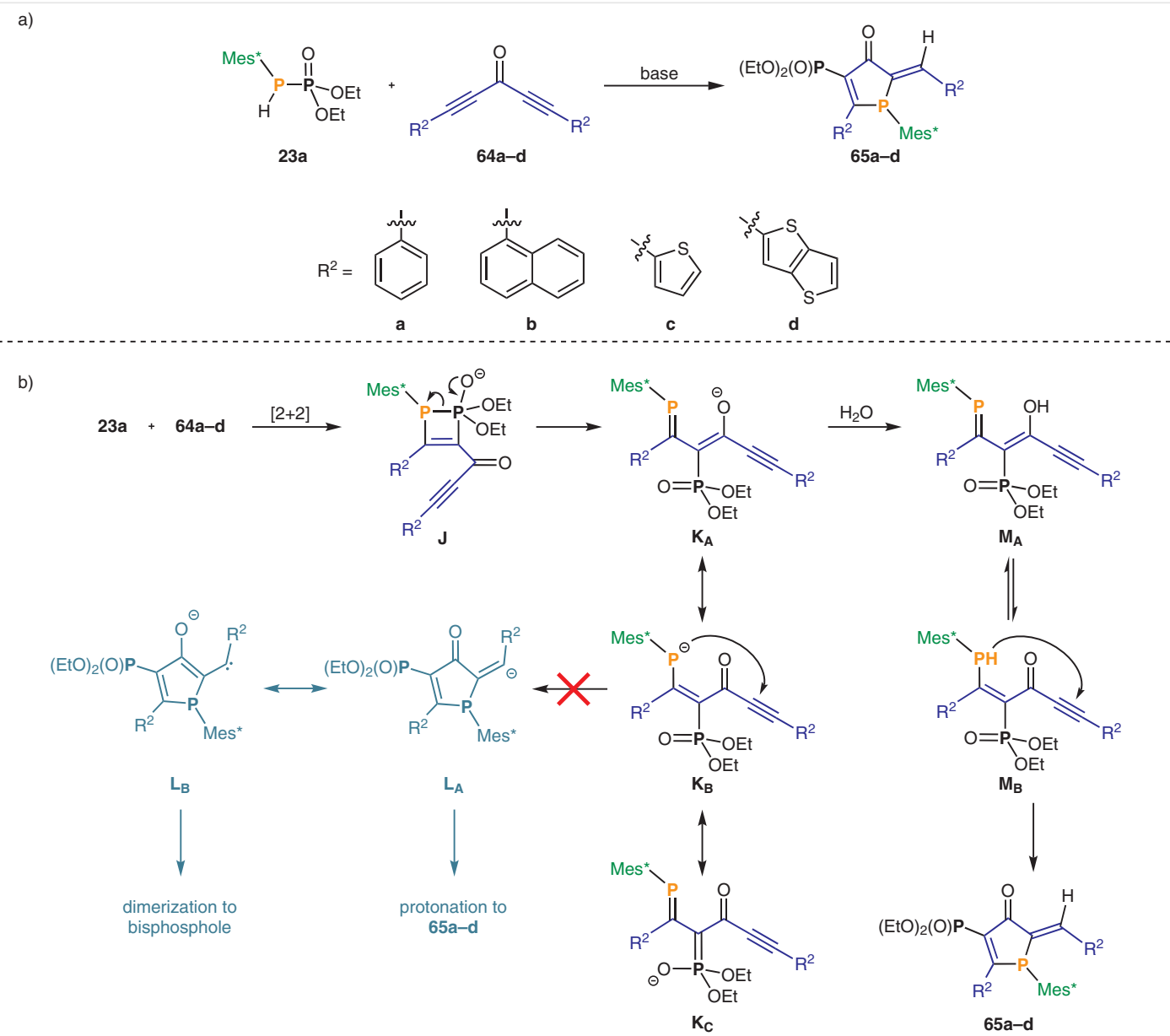

Scheme 11 (a) Reaction of metal-free phosphanylphosphonate 23a with symmetric diacetylenic ketones to form phospholones. (b) Proposed mechanism for the formation of phospholones. ${ }^{49 c}$ 


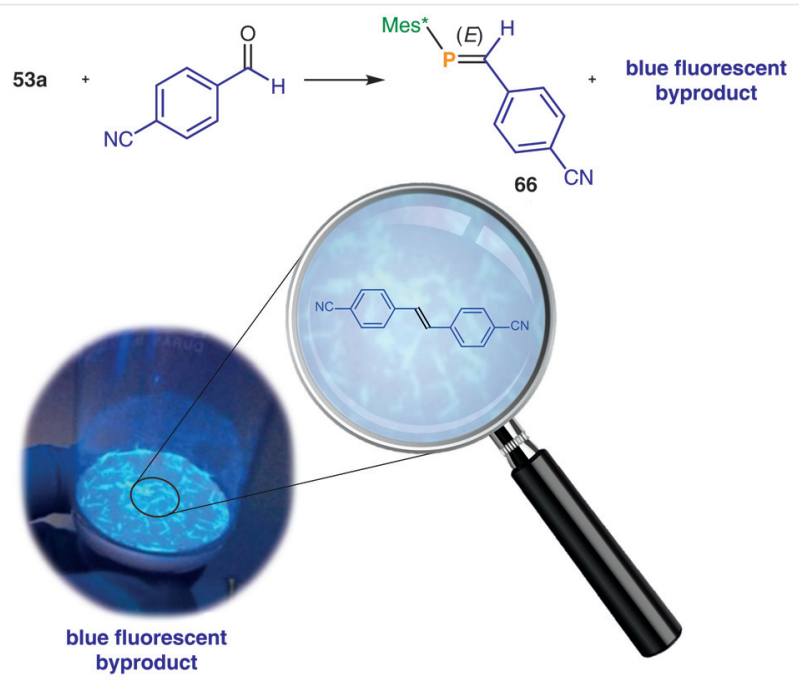

Scheme 12 Phospha-HWE reaction of phosphanylphosphonate 53a with 4-cyanobenzaldehyde and the discovery of the trans-stilbene byproduct as a blue-fluorescing crystalline solid

It was clear that the only possible origin of this stilbene product is the 4-cyanobenzaldehyde, two equivalents of which must have been coupled reductively under the basic reaction conditions to form the alkene. This discovery moved the focus of our whole research group away from $\pi$ conjugated systems to the application of phosphanylphosphonates as reagents for the reductive cross-coupling of carbonyl compounds to alkenes. ${ }^{52}$ The dramatic redirection of the research program was justified by the fact that the observed reactivity, if generally applicable, would constitute a much-needed alternative to the McMurry coupling, the only versatile method for converting carbonyl compounds into alkenes that had been reported in the literature. ${ }^{53}$

We hypothesized that during the aqueous workup of the phospha-HWE reaction, hydroxide ions might attack the electrophilic P-center of phosphaalkene 66, leading to the trivalent phosphinous acid 67, which is known to exist in equilibrium with its phosphine oxide tautomer 68. ${ }^{54}$ The secondary phosphine oxide $\mathbf{6 8}$ resembles classic HornerWittig olefination reagents and, as such, should react with another equivalent of the aldehyde to produce the olefinic product and the corresponding phosphinate byproduct $\mathbf{7 0}$ (Scheme 13).

\section{E-Alkenes by the Reductive Coupling of Two Aldehydes}

The proposed reaction sequence discussed above was probed by ${ }^{31} \mathrm{P}$ NMR spectroscopy, which confirmed the mechanistic model. The coupling proceeds as a one-pot re-

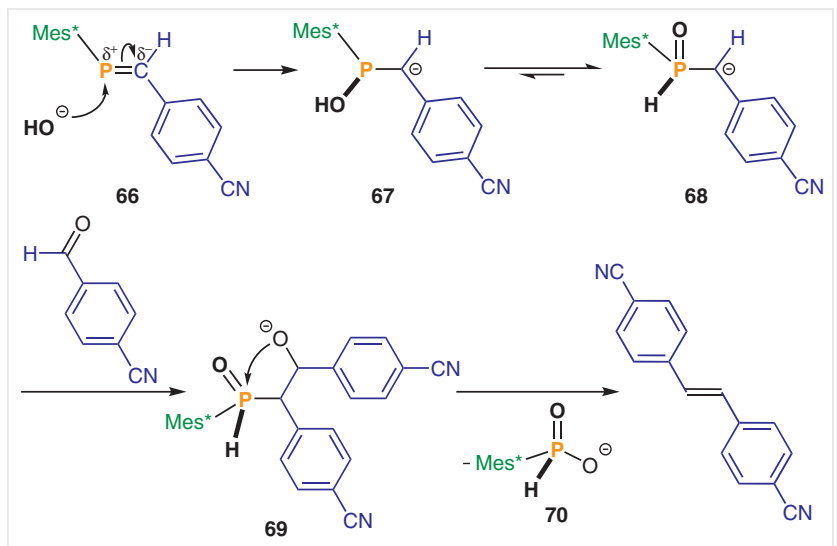

Scheme 13 Proposed mechanistic sequence for the alkene formation by activation of a phosphaalkene with a hydroxide nucleophile and subsequent reaction with an aromatic aldehyde

action, but can mechanistically be dissected into three different stages (Scheme 14). The first stage is a conventional phospha-HWE reaction that converts a first aldehyde into a phosphaalkene by using phosphanylphosphonate 53a as the reagent. Stage 3 is very much related to Horner-Wittigtype chemistry, in which high-valent $\mathrm{P}(\mathrm{V})$ species are used for the olefination of carbonyl compounds. The ingenious aspect of the overall process is its fusion of the chemistry of $\mathrm{P}(\mathrm{III})$, i.e. phosphaalkenes, with that of $\mathrm{P}(\mathrm{V})$ species, as performed in an activation step in stage 2. It is important to note the inversion of the polarity (Umpolung) of the carbonyl carbon atom in the aldehydes during the formation of the phosphaalkenes, which allows the successive coupling chemistry to proceed through an ionic mechanism.

The elucidation of the reaction mechanism allowed us to adjust the reaction parameters and to develop reliable methodologies for the direct formation of various alkenes from two aldehydes. In essence, two different methodologies have been developed. ${ }^{52,55}$

In the initial procedure, $\mathrm{Bu}_{4} \mathrm{NOH}$ was used as the activating agent (Scheme 14, path $\mathrm{A}$ ) to couple two equivalents of benzaldehydes reductively to form the corresponding symmetric alkenes (Table 2). It turned out that the substrate scope in this procedure is rather limited, and only activated (i.e., electron-deficient) benzaldehydes could be coupled efficiently. Nevertheless, these couplings were the first reports of reductive carbonyl couplings by an ionic mechanism. More interesting, though, as alkene formation according to the mechanism in Scheme 14 occurs in a stepwise manner, the possibility of selectively forming nonsymmetric alkenes from two different aldehyde starting materials presented itself. Such site-selectivity in reductive carbonyl coupling chemistry is not possible with the radical mechanism of the McMurry coupling. 


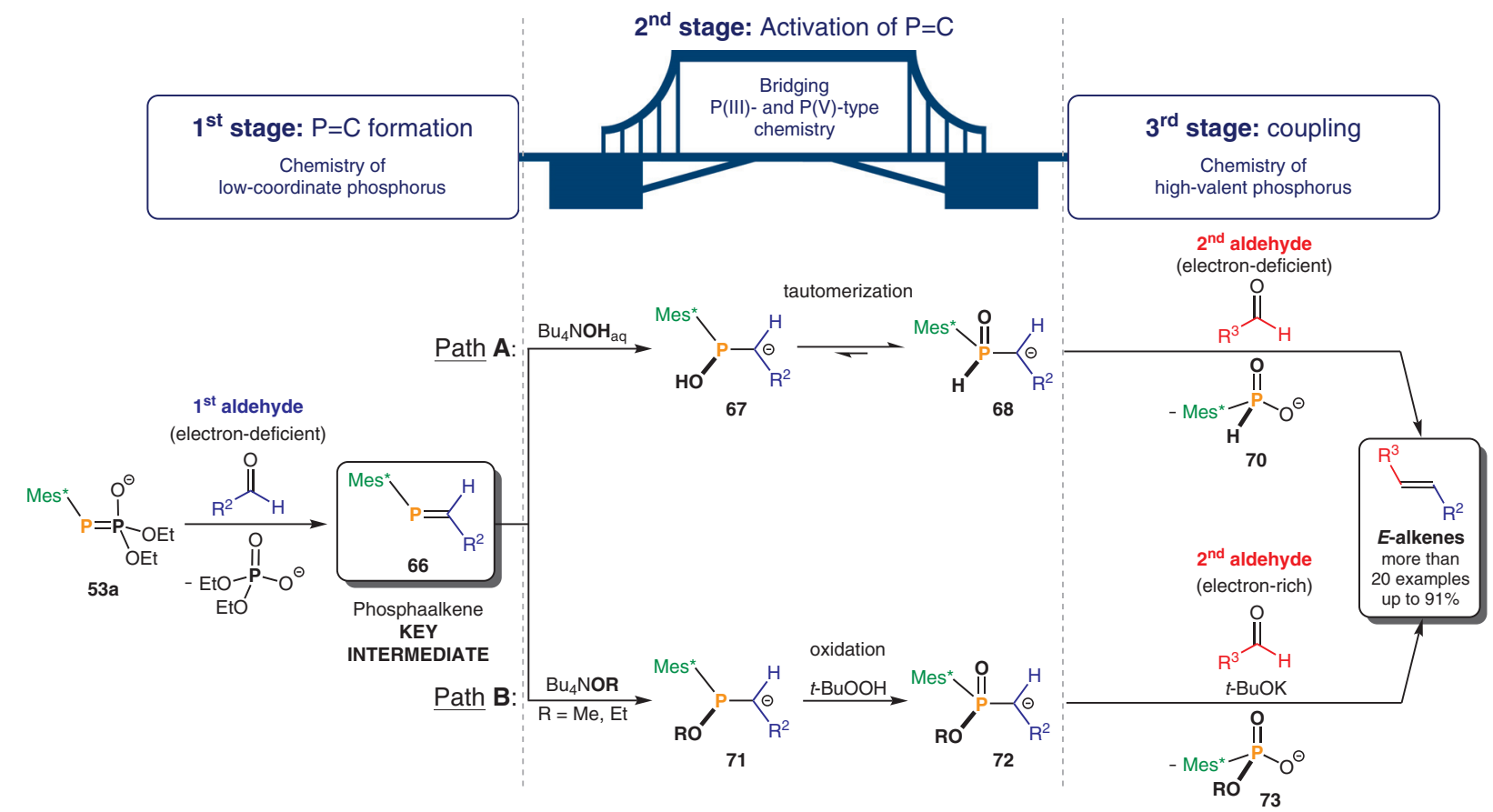

Scheme 14 Summarized reaction pathway for the reductive coupling of two aldehydes via phosphaalkene intermediates. Path A: For electron-deficient second aldehydes. Path B: For electron-rich second aldehydes. The one-pot reaction is a series of three successive steps: first, formation of phosphaalkene; secondly, activation of the $\mathrm{P}=\mathrm{C}$ bond; and thirdly, coupling with a second aldehyde.

Table 2 Reductive Aldehyde Homocouplings To Form Symmetric 1,2Disubstituted E-Alkenes According to Reaction Path $A^{\text {a }}$

\begin{tabular}{lll}
\hline Aldehyde $\quad$ Product & $\begin{array}{l}\text { Conversion } \\
\text { (Isolated yield) (\%) }\end{array}$ \\
\hline
\end{tabular}<smiles>O=Cc1ccccc1</smiles><smiles>N#Cc1ccc(/C=C/c2ccc(C#N)cc2)cc1</smiles><smiles>O=Cc1ccc(Br)cc1</smiles><smiles>Brc1ccc(/C=C/c2ccc(Br)cc2)cc1</smiles><smiles>O=Cc1cccc(Br)n1</smiles>

80

\footnotetext{
a Reaction conditions: ${ }^{52}$ (1) phosphanylphosphonate 23a, LDA, THF, $20^{\circ} \mathrm{C}$, aldehyde (2 equiv), 5 min; (2) 40 wt\% aq $\mathrm{Bu}_{4} \mathrm{NOH}$.

${ }^{b}$ Conversions were determined by ${ }^{1} \mathrm{H}$ NMR spectroscopy with an internal standard; isolated yields are shown in parentheses.
}

The site-selective coupling of two different aldehydes to give nonsymmetric alkenes was first performed by following path A in Scheme 14. Thus, electron-deficient aldehydes, such as 4-cyanobenzaldehyde or 4-bromobenzaldehyde, that performed well as the first aldehydes in the homocoupling experiments were coupled to a variety of (hetero)aromatic aldehydes as well as aliphatic or vinylic aldehydes (Table 3$)^{52}$

Table 3 Nonsymmetric E-Alkenes from the Reductive Coupling of Two

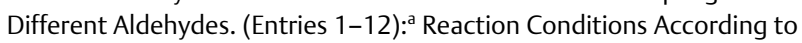
Path A:52 Entries 13-19: ${ }^{b}$ Reaction Conditions According to Path $B^{55}$

\begin{tabular}{|c|c|c|c|}
\hline Entry & First aldehyde $\left(R^{2}\right)$ & Second aldehyde $\left(R^{3}\right)$ & $\begin{array}{l}\text { Conversionc } \\
\text { (Isolated yield) (\%) }\end{array}$ \\
\hline 1 & & & $91(72)$ \\
\hline 2 & & & $56(47)$ \\
\hline 3 & & & 57 \\
\hline 4 & & & 58 \\
\hline 5 & & & 74 \\
\hline
\end{tabular}




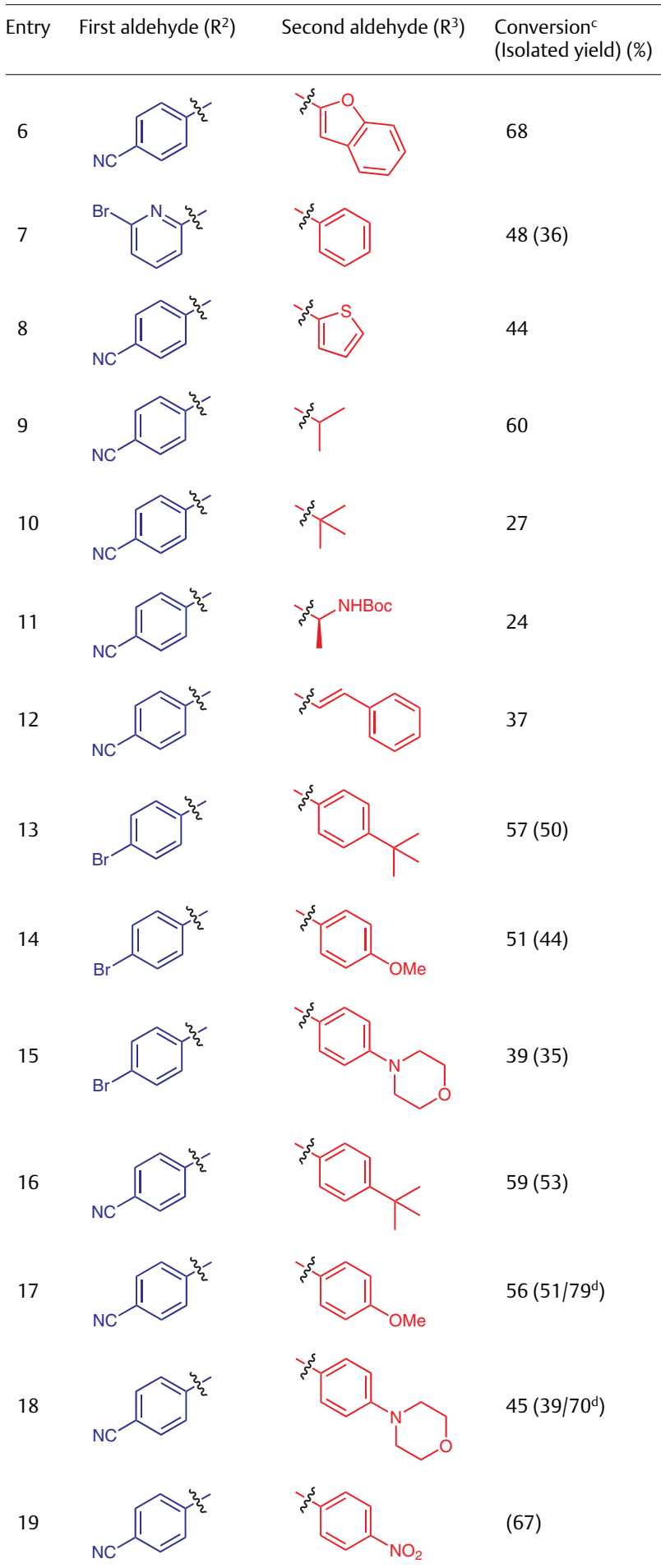

a Reaction conditions: ${ }^{52}$ (1) phosphanylphosphonate 23a, LDA, THF, $20^{\circ} \mathrm{C}$, first aldehyde, 5 min; (2) $40 \mathrm{wt} \%$ aq $\mathrm{Bu}_{4} \mathrm{NOH}$, second aldehyde.

b Reaction conditions: ${ }^{55}$ phosphanylphosphonate 23a, LDA, THF, $20^{\circ} \mathrm{C}$, first aldehyde, 5 min; (2) $\mathrm{Bu}_{4} \mathrm{NOMe}(20 \mathrm{wt} \%$ in $\mathrm{MeOH}), 30$ min; (3) $t-\mathrm{BuOOH}, 5-$ 6 hours; (4) $t$-BuOK, second aldehyde.

${ }^{\mathrm{C}}$ Conversions were determined by ${ }^{1} \mathrm{H}$ NMR spectroscopy with an internal standard; isolated yields appear in brackets.

${ }^{d}$ The coupling was performed with 0.5 equivalents of the second aldehyde as a limiting reactant.
Notably, the conversions and yields for couplings between electron-deficient aldehydes are generally high. Electron-rich aldehydes, however, react more sluggishly under these conditions, and only trace amounts of products are observed. To address this limitation in substrate scope, a modification of the protocol was developed. We rationalized that an additional alkoxide substituent on the $P$-center of the intermediate after the activation step would enhance the electropositive character of the $P$-atom. The resulting phosphinate intermediate $\mathbf{7 2}$ should be more reactive than phosphine oxide $\mathbf{6 8}$ due to the increased nucleophilicity of its deprotonated form (Scheme 14, path B). ${ }^{55}$ The synthetic approach for this was to use a solution of tetrabutylammonium methoxide or ethoxide, and to oxidize the transient phosphinite 71 prior to the coupling with the second aldehyde. The modified conditions (Scheme 14, Path B) allowed the coupling of electron-deficient aldehydes with electronrich (deactivated) aldehydes, giving a direct access to stilbenes with interesting push-pull electronic properties (Table 3, entries 13-19). From Table 3, it is also clear that the substrate scope for the second aldehyde is much larger than that of the first. As described in Section 3.3 (Table 1), phosphaalkene formation generally proceeds well for most aldehydes. ${ }^{46}$ In case the phosphaalkenes contain electron-donating C-substituents, the electrophilicity of the P-center is, however, rather low, which results in an inertness of $\mathbf{6 6}$ towards hydroxide attack. Even if the phosphine oxide $\mathbf{6 8}$ is formed, electron-rich substituents $\mathrm{R}^{2}$ lower the acidity of the $\alpha$-protons, making electron-rich aldehydes unsuitable as the first substrates.

With the aim to overcome this limitation and to further broaden the substrate scope, our group examined a new approach starting with a phospha-Peterson reaction to form a phosphaalkenes in stage one. ${ }^{56}$ This approach permits the preparation of intermediate phosphaalkenes with a sterically less-demanding mesitylene (Mes) protecting group. These phosphaalkenes are transformed in situ into the corresponding phosphinates, which exhibit a high reactivity even with electron-rich C-substituents, thereby allowing the coupling of two deactivated aldehydes. Recent work has reported the coupling of ketones and benzaldehydes to trisubstituted alkenes by using $\mathrm{PhP}(\mathrm{TMS}) \mathrm{Li}$ as a coupling reagent. ${ }^{57}$

All coupling protocols via phosphaalkene intermediates use inexpensive feedstock aldehydes directly in a one-pot reaction to form nonsymmetric alkenes. This is not the case in classic carbonyl olefination methods, such as the Wittig, HWE, Julia-Kocienski, and Peterson reactions, where multistep syntheses are required to prepare highly specific reagents. The coupling reactions that we have developed over the last three years typically work under mild conditions at room temperature within short times, are free of transition metals, and follow a sequential ionic mechanism that offers controlled access to dissimilarly substituted alkenes from two different aldehyde substrates. 


\section{Conclusions and Outlook}

This account summarized the group's journey into phosphanylphosphonates over the last ten years. The entry into this area came from the simple idea of introducing low-coordinate phosphorus in the form of phosphaalkenes into acetylene scaffolds. In comparison to all-carbon-based oligoenynes, acetylenic phosphaalkenes offer interesting optoelectronic properties and possibilities for post-synthetic modification owing to the free electron pair at the phosphorus atom. The synthetic approaches to such systems, however, are limited, and while seeking for alternative routes we encountered the phospha-Horner-Wadsworth-Emmons reaction. This reaction uses phosphanylphosphonates to prepare phosphaalkenes from aldehydes and ketones. New synthetic protocols for metal-coordinated reagents and the first metal-free reagents were developed, starting from simple dichlorophosphine precursors. Moreover, we were able to propose a plausible mechanism for the phospha-HWE reaction based on crystallographically characterized reaction intermediates. The application of the phosphanylphosphonates in reactions with acetylenic ketones revealed a complex product outcome, depending on the type of acetylene substituents. Whereas tungstencoordinated reagents yield 1,2-oxaphospholes, cumulenes, and bisphospholes, the use of the metal-free reagent results in the formation of phospholones.

The work on phosphaalkenes led to the discovery that phosphanylphosphonates can promote reductive aldehydealdehyde coupling. This reaction occurs through a phosphaalkene intermediate that is formed in a phospha-HWE reaction from the phosphanylphosphonate and the first aldehyde. Upon phosphaalkene activation, the olefination reagent formed in situ converts the second aldehyde into a nonsymmetric alkene. Extensions of this concept to an even broader substrate scope, as well as the development of reagents for the formation of $Z$-alkenes, are currently ongoing in our laboratory.

\section{Funding Information}

We gratefully acknowledge the funding supplied by Uppsala University and the Swedish Research Council for our group's activities in phosphaorganic chemistry.

\section{Acknowledgment}

S.O. wishes to acknowledge the contributions made by all our coworkers to the group's program on phosphaorganic chemistry. Without their energy and dedication, this work would not have been possible. The names of the co-workers can be found in the respective citations.

\section{References}

(1) Nielsen, M. B.; Diederich, F. Chem. Rev. 2005, 105, 1837.

(2) (a) Maier, M. E. Synlett 1995, 3. (b) Nicolaou, K. C.; Dai, W.-M. Angew. Chem. Int. Ed. 1991, 30, 1387. (c) Smith, A. L.; Nicolaou, K. C. J. Med. Chem. 1996, 39, 2103.

(3) Acetylene Chemistry: Chemistry, Biology, and Material Science; Diederich, F.; Stang, P. J.; Tykwinski, R. R., Eds.; Wiley-VCH: Weinheim, 2005.

(4) (a) Nielsen, M. B.; Diederich, F. Synlett 2002, 544. (b) Nielsen, M. B.; Diederich, F. Chem. Rec. 2002, 2, 189. (c) Martin, R. E.; Mäder, T.; Diederich, F. Angew. Chem. Int. Ed. 1999, 38, 817.

(5) (a) Bunz, U. H. F. Chem. Rev. 2000, 100, 1605. (b) Nielsen, N. B.; Diederich, F. In Modern Arene Chemistry; Astruc, D., Ed.; VCHWiley: Weinheim, 2002, 196. (c) Petty, M. C. Organic and Molecular Electronics: From Principles to Practice, 2nd ed; Wiley: Chichester, 2019.

(6) (a) Haley, M. M.; Martín, N.; Würthner, F. Org. Chem. Front. 2017, 4, 648. (b) Carbon Rich Compounds II: Macrocyclic Oligoacetylenes and Other Linearly Conjugated Systems; de Meijere, A., Ed.; Springer: Berlin, 1999. (c) Conjugated Polymers and Related Materials: Macrocyclic Oligoacetylenes and Other Linearly Conjugated Systems: The Interconnection of Chemical and Electronic Structure: Proceedings of the Eighty-First Nobel Symposium (Luleå, Sweden, 1991); Salaneck, W. R.; Lundström, I.; Rånby, B., Eds.; Oxford University Press: Oxford, 1993. (d) Handbook of Conducting Polymers, 4th ed; Reynolds, J. R.; Thompson, B. C.; Skotheim, T. A., Ed.; CRC Press: Boca Raton, 2019. (e) CarbonRich Compounds: From Molecules to Materials; Haley, M. M.; Rik, R. T., Ed.; Wiley-VCH: Weinheim, 2006. (f) Kraft, A.; Grimsdale, A. C.; Holmes, A. B. Angew. Chem. Int. Ed. 1998, 37, 402. (g) Kivala, M.; Diederich, F. Acc. Chem. Res. 2009, 42, 235. (h) Frampton, M. J.; Anderson, H. L. Angew. Chem. Int. Ed. 2007, 46, 1028. (i) Robertson, N.; McGowan, C. A. Chem. Soc. Rev. 2003, 32, 96. (j) Elbing, M.; Ochs, R.; Koentopp, M.; Fischer, M.; von Hänisch, C.; Weigend, F.; Evers, F.; Weber, H. B.; Mayor, M. Proc. Natl. Acad. Sci. U.S.A. 2005, 102, 8815.

(7) Dillon, K. B.; Mathey, F.; Nixon, J. F. Phosphorus: The Carbon Copy: From Organophosphorus to Phospha-Organic Chemistry; Wiley: Chichester, 1998.

(8) Mathey, F. Angew. Chem. Int. Ed. 2003, 42, 1578.

(9) Pauling, L. The Nature of the Chemical Bond and the Structure of Molecules and Crystals: An Introduction to Modern Structural Chemistry, 3rd ed; Cornell University Press: Ithaca, 1960, 88.

(10) (a) Waluk, J.; Klein, H. P.; Ashe, A. J.; Michl, J. Organometallics 1989, 8, 2804. (b) Schoeller, W. W. J. Chem. Soc., Chem. Commun. $1985,334$.

(11) (a) Appel, R. In Multiple Bonds and Low Coordination in Phosphorus Chemistry: Phosphaalkenes, Phosphacarbaoligoenes, and Phosphaallenes; Regitz, M.; Scherer, O. J., Eds.; Thieme: Stuttgart, 1990, 157. (b) Marinetti, A.; Ricard, L.; Mathey, F. Synthesis 1992, 157. (c) Gates, D. P. In New Aspects in Phosphorus Chemistry V; Majoral, J.-P., Ed.; Springer: Berlin, 2005, 107. (d) Bates, J. I.; Dugal-Tessier, J.; Gates, D. P. Dalton Trans. 2010, 39, 3151. (e) Pietschnig, R.; Orthaber, A. In Reference Module in Chemistry, Molecular Sciences and Chemical Engineering; Elsevier: Amsterdam, 2016, DOI:/10.1016/B978-0-12-409547-2.11721-4. (f) Quin, L. D. A Guide to Organophosphorus Chemistry; WileyInterscience: New York, 2000.

(12) (a) Nauroozi, D.; Orthaber, A. Eur. J. Inorg. Chem. 2016, 2016, 709. (b) Simpson, M. C.; Protasiewicz, J. D. Pure Appl. Chem. 2013, 85, 801.

(13) Le Floch, P. Coord. Chem. Rev. 2006, 250, 627. 
(14) Weber, L. Eur. J. Inorg. Chem. 2000, 2000, 2425.

(15) (a) Baumgartner, T.; Réau, R. Chem. Rev. 2006, 106, 4681. (b) Shameem, M. A.; Orthaber, A. Chem. Eur. J. 2016, 22, 10718.

(16) Schäfer, B.; Öberg, E.; Kritikos, M.; Ott, S. Angew. Chem. Int. Ed. 2008, 47, 8228.

(17) (a) van der Sluis, M.; Klootwijk, A.; Wit, J. B. M.; Bickelhaupt, F.; Veldman, N.; Spek, A. L.; Jolly, P. W. J. Organomet. Chem. 1997, 529, 107. (b) Appel, R.; Casser, C.; Knoch, F. Chem. Ber. 1984, 117, 2693.

(18) Yoshifuji, M. J. Organomet. Chem. 2000, 611, 210.

(19) (a) Goede, S. J.; Bickelhaupt, F. Chem. Ber. 1991, 124, 2677. (b) Orthaber, A.; Öberg, E.; Jane, R. T.; Ott, S. Z. Anorg. Allg. Chem. 2012, 638, 2219.

(20) Romanenko, V. D.; Sanchez, M.; Sarina, T. V.; Mazières, M.-R.; Wolf, R. Tetrahedron Lett. 1992, 33, 2981.

(21) Jun, H.; Young, V. G. Jr; Angelici, R. J. Organometallics 1994, 13, 2444.

(22) Öberg, E.; Schäfer, B.; Geng, X.-L.; Pettersson, J.; Hu, Q.; Kritikos, M.; Rasmussen, T.; Ott, S. J. Org. Chem. 2009, 74, 9265.

(23) (a) Reich, H. J.; Holladay, J. E. Angew. Chem. Int. Ed. 1996, 35, 2365. (b) Reich, H. J.; Holladay, J. E.; Walker, T. G.; Thompson, J. L. J. Am. Chem. Soc. 1999, 121, 9769.

(24) Geng, X.-L.; Ott, S. Chem. Commun. 2009, 7206.

(25) Öberg, E.; Ott, S. Phosphorus, Sulfur Silicon Relat. Elem. 2013, $188,164$.

(26) Shameem, M. A.; Esfandiarfard, K.; Öberg, E.; Ott, S.; Orthaber, A. Chem. Eur. J. 2016, 22, 10614.

(27) (a) Geng, X.-L.; Hu, Q.; Schäfer, B.; Ott, S. Org. Lett. 2010, 12, 692. (b) Geng, X.-L.; Ott, S. Chem. Eur. J. 2011, 17, 12153. (c) Öberg, E.; Geng, X.-L.; Santoni, M.-P.; Ott, S. Org. Biomol. Chem. 2011, 9, 6246.

(28) Svyaschenko, Y. V.; Orthaber, A.; Ott, S. Chem. Eur. J. 2016, 22, 4247.

(29) Zhao, Y.; McDonald, R.; Tykwinski, R. R. J. Org. Chem. 2002, 67, 2805.

(30) Martin, R. E.; Wytko, J. A.; Diederich, F.; Boudon, C.; Gisselbrecht, J.-P.; Gross, M. Helv. Chim. Acta 1999, 82, 1470.

(31) Zhao, Y.; Zhou, N.; Slepkov, A. D.; Ciulei, S. C.; McDonald, R.; Hegmann, F. A.; Tykwinski, R. R. Helv. Chim. Acta 2007, 90, 909.

(32) (a) Eisler, S.; Tykwinski, R. R. Angew. Chem. Int. Ed. 1999, 38, 1940. (b) Zhao, Y.; Ciulei, S. C.; Tykwinski, R. R. Tetrahedron Lett. 2001, 42, 7721.

(33) Bruschi, M.; Giuffreda, M. G.; Lüthi, H. P. Chem. Eur. J. 2002, 8, 4216.

(34) Shah, S.; Protasiewicz, J. D. Coord. Chem. Rev. 2000, 210, 181.

(35) (a) Le Floch, P.; Marinetti, A.; Ricard, L.; Mathey, F. J. Am. Chem. Soc. 1990, 112, 2407. (b) Le Floch, P.; Mathey, F. Synlett 1990, 171.

(36) (a) Shah, S.; Concolino, T.; Rheingold, A. L.; Protasiewicz, J. D. Inorg. Chem. 2000, 39, 3860. (b) Shah, S.; Protasiewicz, J. D. Chem. Commun. 1998, 1585.

(37) (a) Marinetti, A.; Mathey, F. Angew. Chem., Int. Ed. Engl. 1988, 27, 1382. (b) Marinetti, A.; Bauer, S.; Ricard, L.; Mathey, F. Organometallics 1990, 9, 793. (c) Marinetti, A.; Ricard, L.; Mathey, F. Organometallics 1990, 9, 788.
(38) Marinetti, A.; Mathey, F. Tetrahedron 1989, 45, 3061.

(39) Bauer, S.; Marinetti, A.; Mathey, F. Heteroat. Chem. 1991, 2, 277.

(40) Arkhypchuk, A. I.; Santoni, M.-P.; Ott, S. Organometallics 2012, $31,1118$.

(41) De Vaumas, R.; Marinetti, A.; Ricard, L.; Mathey, F. J. Am. Chem. Soc. 1992, 114, 261.

(42) Mathey, F.; Marinetti, A.; Bauer, S.; Le Floch, P. Pure Appl. Chem. 1991, 63, 855 .

(43) (a) Maryanoff, B. E.; Reitz, A. B. Chem. Rev. 1989, 89, 863. (b) Edmonds, M.; Abell, A. In Modern Carbonyl Olefinations; Takeda, T., Ed.; Wiley-VCH: Weinheim, 2003, 1. (c) Appel, R.; Loos, R.; Mayr, H. J. Am. Chem. Soc. 2009, 131, 704.

(44) Arkhypchuk, A. I.; Svyaschenko, Y. V.; Orthaber, A.; Ott, S. Angew. Chem. Int. Ed. 2013, 52, 6484.

(45) Smith, R. C.; Protasiewicz, J. D. J. Am. Chem. Soc. 2004, 126, 2268.

(46) Esfandiarfard, K.; Arkhypchuk, A. I.; Orthaber, A.; Ott, S. Dalton Trans. 2016, 45, 2201.

(47) (a) Cowley, A. H.; Kilduff, J. E.; Newman, T. H.; Pakulski, M. J. Am. Chem. Soc. 1982, 104, 5820. (b) Issleib, K.; Schmidt, H.; Wirkner, C. Z. Anorg. Allg. Chem. 1982, 488, 75.

(48) (a) Couret, C.; Escudie, J.; Ranaivonjatovo, H.; Satge, J. Organometallics 1986, 5, 113. (b) Märkl, G.; Kreitmeier, P. Angew. Chem. Int. Ed. 1988, 27, 1360 .

(49) (a) Arkhypchuk, A. I.; Orthaber, A.; Mihali, V. A.; Ehlers, A.; Lammertsma, K.; Ott, S. Chem. Eur. J. 2013, 19, 13692. (b) Arkhypchuk, A. I.; Santoni, M.-P.; Ott, S. Angew. Chem. Int. Ed. 2012, 51, 7776. (c) Obermeier, M.; Arkhypchuk, A. I. J. Org. Chem. 2019, 84, 3491.

(50) Arkhypchuk, A. I.; Mijangos, E.; Lomoth, R.; Ott, S. Chem. Eur. J. 2014, 20, 16083.

(51) (a) Brown, G. W. J. Chem. Soc. C 1967, 2018. (b) Braña, M. F.; Morán, M.; de Vega, M. J. P.; Pita-Romero, I.; Walker, N. Tetrahedron 1995, 51, 9127. (c) Barluenga, J.; Lopez, F.; Palacios, F.; Sánchez-Ferrando, F. Tetrahedron Lett. 1988, 29, 381.

(52) Esfandiarfard, K.; Mai, J.; Ott, S. J. Am. Chem. Soc. 2017, 139, 2940.

(53) (a) Ephritikhine, M.; Villiers, C. In Modern Carbonyl Olefination; Takeda, T., Ed.; Wiley-VCH: Weinheim, 2003, 223. (b) McMurry, J. E. Chem. Rev. 1989, 89, 1513. (c) McMurry, J. E.; Fleming, M. P. J. Am. Chem. Soc. 1974, 96, 4708.

(54) (a) Hoge, B.; Bader, J.; Beckers, H.; Kim, Y. S.; Eujen, R.; Willner, H.; Ignatiev, N. Chem. Eur. J. 2009, 15, 3567. (b) Hoge, B.; Garcia, P.; Willner, H.; Oberhammer, H. Chem. Eur. J. 2006, 12, 3567. (c) Hoge, B.; Neufeind, S.; Hettel, S.; Wiebe, W.; Thösen, C. J. Organomet. Chem. 2005, 690, 2382. (d) Kurscheid, B.; Wiebe, W.; Neumann, B.; Stammler, H.-G.; Hoge, B. Eur. J. Inorg. Chem. 2011, 2011, 5523. (e) Bloomfield, A. J.; Qian, J. M.; Herzon, S. B. Organometallics 2010, 29, 4193.

(55) Mai, J.; Arkhypchuk, A. I.; Gupta, A. K.; Ott, S. Chem. Commun. 2018, 54, 7163.

(56) Arkhypchuk, A. I.; D'Imperio, N.; Ott, S. Org. Lett. 2018, 20, 5086.

(57) Arkhypchuk, A. I.; D'Imperio, N.; Ott, S. Chem. Commun. 2019, 55,6030 . 